\title{
Motion Estimation and Signaling Techniques for 2D+t Scalable Video Coding
}

\author{
M. Tagliasacchi, D. Maestroni, S. Tubaro, and A. Sarti \\ Dipartimento di Elettronica e Informazione, Politecnico di Milano, Piazza Leonardo da Vinci, 3220133 Milano, Italy
}

Received 1 March 2005; Revised 5 August 2005; Accepted 12 September 2005

\begin{abstract}
We describe a fully scalable wavelet-based $2 \mathrm{D}+\mathrm{t}$ (in-band) video coding architecture. We propose new coding tools specifically designed for this framework aimed at two goals: reduce the computational complexity at the encoder without sacrificing compression; improve the coding efficiency, especially at low bitrates. To this end, we focus our attention on motion estimation and motion vector encoding. We propose a fast motion estimation algorithm that works in the wavelet domain and exploits the geometrical properties of the wavelet subbands. We show that the computational complexity grows linearly with the size of the search window, yet approaching the performance of a full search strategy. We extend the proposed motion estimation algorithm to work with blocks of variable sizes, in order to better capture local motion characteristics, thus improving in terms of rate-distortion behavior. Given this motion field representation, we propose a motion vector coding algorithm that allows to adaptively scale the motion bit budget according to the target bitrate, improving the coding efficiency at low bitrates. Finally, we show how to optimally scale the motion field when the sequence is decoded at reduced spatial resolution. Experimental results illustrate the advantages of each individual coding tool presented in this paper. Based on these simulations, we define the best configuration of coding parameters and we compare the proposed codec with MC-EZBC, a widely used reference codec implementing the $\mathrm{t}+2 \mathrm{D}$ framework.
\end{abstract}

Copyright ( 2006 Hindawi Publishing Corporation. All rights reserved.

\section{INTRODUCTION}

Today's video streaming applications require codecs to provide a bitstream that can be flexibly adapted to the characteristics of the network and the receiving device. Such codecs are expected to fulfill the scalability requirements so that encoding is performed only once, while decoding takes place each time at different spatial resolutions, frame rates, and bitrates. Consider for example streaming a video content to TV sets, PDAs, and cellphones at the same time. Obviously each device has its own constraints in terms of bandwidth, display resolution, and battery life. For this reason it would be useful for the end users to subscribe to a scalable video stream in such a way that a representation of the video content matching the device characteristics can be extracted at decoding time. Wavelet-based video codecs have proved to be able to naturally fit this application scenario, by decomposing the video sequence into a plurality of spatio-temporal subbands. Combined with an embedded entropy coding of wavelet coefficients such as JPEG2000 [1], SPIHT (set partitioning in hierarchical trees) [2], EZBC (embedded zeroblock coding) [3], or ESCOT (motion-based embedded subband coding with optimized truncation) [4], it is possible to support spatial, temporal, and SNR (signal-to-noise ratio) scalability. Broadly speaking, two families of wavelet-based video codecs have been described in the literature:

(i) $t+2 \mathrm{D}$ schemes [5-7]: the video sequence is first filtered in the temporal direction along the motion trajectories (MCTF-motion-compensated temporal filtering [8]) in order to tackle temporal redundancy. Then, a 2D wavelet transform is carried out in the spatial domain. Motion estimation/compensation takes place in the spatial domain, hence conventional coding tools used in nonscalable video codecs can be easily reused;

(ii) $2 \mathrm{D}+\mathrm{t}$ (or in-band) schemes $[9,10]$ : each frame of the video sequence is wavelet-transformed in the spatial domain, followed by MCTF. Motion estimation/compensation is carried out directly in the wavelet domain.

Due to the nonlinear motion warping operator needed in the temporal filtering stage, the order of the transforms does not commute. In fact the wavelet transform is not shiftinvariant and care has to be taken since the motion estimation/compensation task is performed in the wavelet domain. In the literature several approaches have been used to tackle this issue. Although known under different names (low-band-shift [11], ODWT (overcomplete discrete wavelet 
transform) [12], redundant DWT [10]), all the solutions represent different implementations of the algorithm á trous [13], that computes an overcomplete wavelet decomposition by omitting the decimators in the fast DWT algorithm and stretching the wavelet filters by inserting zeros. A two-level ODWT transform on a 1D signal is illustrated in Figure 1, where $H_{0}(z)$ and $H_{1}(z)$ are, respectively, the wavelet low-pass and high-pass filters used in the conventional critically sampled DWT. $H_{i}^{k}(z)$ is the dilated version of $H_{i}(z)$ obtained by inserting $k-1$ zeros between two consecutive samples. The extension to $2 \mathrm{D}$ signals is straightforward with a separable approach. Despite its higher complexity, a $2 \mathrm{D}+\mathrm{t}$ scheme comes with the advantage of reducing the impact of blocking artifacts caused by the failure of block-based motion models. This is because such artifacts are canceled out by the inverse DWT spatial transform, without the need to adopt some sort of deblocking filtering. This fact greatly enhances the perceptual quality of reconstructed sequences, especially at low bitrates. Furthermore, as shown in $[14,15], 2 \mathrm{D}+\mathrm{t}$ approaches naturally fit the spatial scalability requirements providing higher coding efficiency when the sequence is decoded at reduced spatial resolution. This is due to the fact that with in-band motion compensation it is possible to limit the problem of drift that occurs when decoder does not have access to all the wavelet subbands used at the encoder side. Finally, 2D+t schemes naturally support multi-hypothesis motion compensation taking advantage of the redundancy of the ODWT [10].

\subsection{Motivations and goals}

In this paper we present a fully scalable video coding architecture based on a $2 \mathrm{D}+\mathrm{t}$ approach. Our contribution is at the system level. Figure 2 depicts the overall video coding architecture, emphasizing the modules we are focusing on in this paper.

It is widely acknowledged that motion modeling has a fundamental importance in the design of a video coding architecture in order to match the coding efficiency of state-of-the-art codecs. As an example, much of the coding gain observed in the recent H.264/AVC standard [16] is due to more sophisticated motion modeling tools (variable block sizes, quarter-pixel motion accuracy, multiple reference frames, etc). Motion modeling is particularly relevant especially when the sequences are decoded at low bitrates and at reduced spatial resolution, because a significant fraction of the bit budget is usually allocated to describe motion-related information. This fact motivates us to focus our attention on motion estimation/compensation and motion signaling techniques to improve the coding efficiency of the proposed $2 \mathrm{D}+\mathrm{t}$ wavelet-based video codec. While achieving better compression, we also want to keep the computational complexity of the encoder under control, in order to design a practical architecture.

In Section 2, we describe the details of the proposed $2 \mathrm{D}+\mathrm{t}$ scalable video codec (see Figure 2). Based on this coding framework, we propose novel techniques to improve the coding efficiency and reduce the complexity of the encoder.

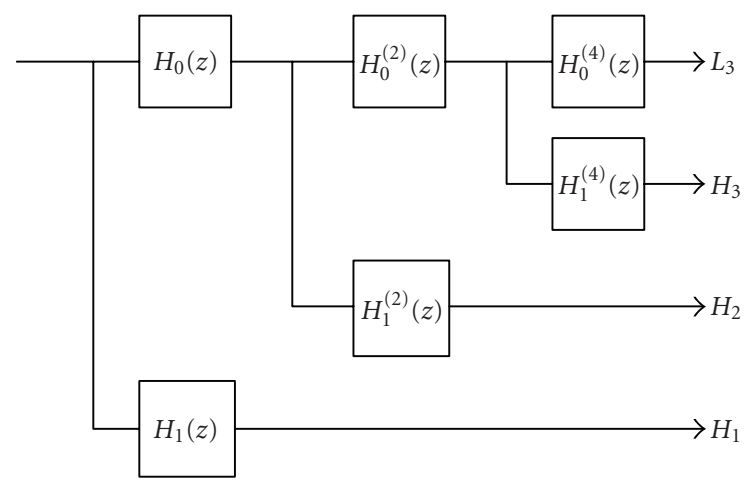

Figure 1: Two level overcomplete DWT (ODWT) of a 1D signal according to the algorithm á trous implementation.

Specifically, we propose the following:

(i) in Section 2.1, a fast motion estimation algorithm that is meant to work in the wavelet domain (FIBME_-fast in-band motion estimation), exploiting the geometrical properties of the wavelet subbands. Section 2.1 elaborates on this topic comparing the computational complexity of the proposed approach with that of an exhaustive full search;

(ii) in Section 2.2, the FIBME algorithm is further extended to work with blocks of variable size;

(iii) in Section 2.3, a scalable representation of the motion model is introduced, which is suitable for variable block sizes and allows to adapt the bit budget allocated to motion according to the target bitrate (see Section 2.3);

(iv) in Section 2.4, a formal analysis describing how the motion field estimated at full resolution can be adapted at reduced spatial resolutions. We show that motion vector truncation, adopted in the reference implementation of the MC-EZBC codec [5], is not the optimal choice when the motion field resolution needs to be scaled.

The paper builds upon our previous work appeared in [17-19].

\subsection{Related works}

In $2 \mathrm{D}+\mathrm{t}$ wavelet-based video codecs, motion estimation/ compensation needs to be carried out directly in the wavelet domain. Although a great deal of works focuses on how to avoid the shift variance of the wavelet transform [9-11, 20], the problem of fast motion estimation in $2 \mathrm{D}+\mathrm{t}$ schemes is not thoroughly investigated in the literature. References [21, 22] propose similar algorithms for in-band motion estimation. In both cases different motion vectors are assigned to each scale of wavelet subbands. In order to decrease the complexity of the motion search, the algorithms work in a multiresolution fashion, in such a way that the motion search at a given resolution is initialized with the estimate obtained at lower resolution. The proposed fast motion estimation 


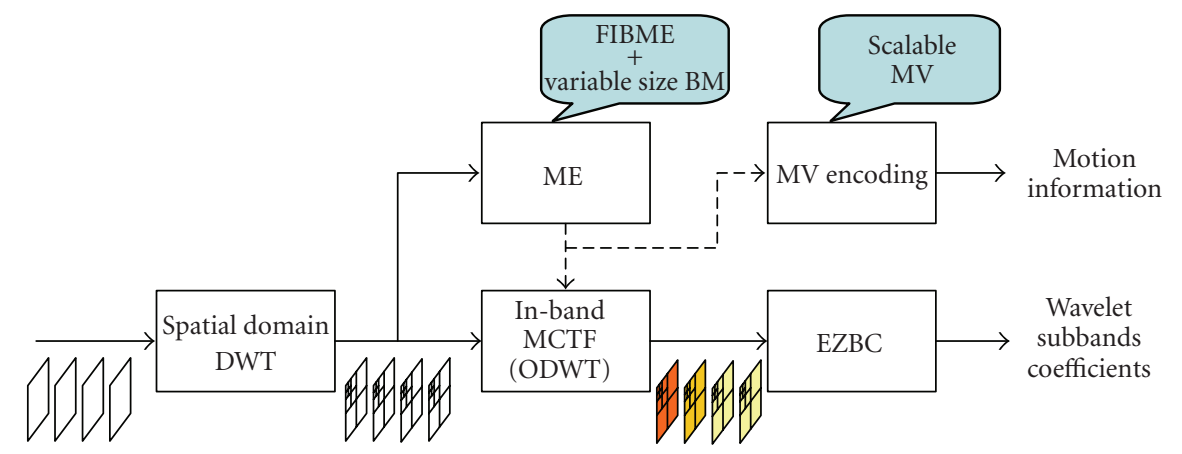

FIGURE 2: Block diagram of the proposed scalable 2D+t coding architecture. Call-outs point to the novel features described in this paper.

algorithm shares the multi-resolution approach of $[21,22]$. Despite this similarity, the proposed algorithm takes full advantage of the geometrical properties of the wavelet subbands, and different motion vectors are used to compensate subbands at the same scale but having different orientation (see Section 2.1), thus giving more flexibility in the modeling of local motion.

Variable size block matching is well known in the literature, at least when it is applied in the spatial domain. The state-of-the-art H.264/AVC [16] standard efficiently exploits this technique. In [23], a hierarchical variable size block matching (HVSBM) algorithm is used in the context of a $\mathrm{t}+2 \mathrm{D}$ wavelet-based codec. The MC-EZBC codec [5] adopts the same algorithm in the motion estimation phase. The authors of [24] independently proposed a variable size block matching strategy within their $2 \mathrm{D}+\mathrm{t}$ wavelet-based codec. The search for the best motion partition is close to the idea of H.264/AVC, since all the possible block partitions are tested in order to determine the optimal one. On the other hand, the algorithm proposed in this paper (see Section 2.2) is more similar to the HVSBM algorithm [23], as the search is suboptimal but faster.

Scalability of motion vector was first proposed in [25] and later further discussed in [26], where JPEG2000 is used to encode the motion field components. The work in [26] assumes that fixed block sizes (or regular meshes) are used in the motion estimation phase. More recently, other works have appeared in the literature [27-29], describing coding algorithms for motion fields having arbitrary block sizes specifically designed for wavelet-based scalable video codecs. The algorithm described in this paper has been designed independently and shares the general approach of $[26,27]$, since the motion field is quantized when decoding at low bitrates. Despite these similarities, the proposed entropy coding scheme is novel and it is inspired to SPIHT [2], allowing lossy to lossless representation of the motion field (see Section 2.3).

\section{PROPOSED 2D+t CODEC}

Figure 2 illustrates the functional modules that compose the proposed $2 \mathrm{D}+\mathrm{t}$ codec. First, a group of pictures (GOP) is fed in input and each frame is wavelet transformed in the spatial domain using Daubechies 9/7 filters. Then, in-band MCTF is performed using the redundant representation of the ODWT to combat shift variance. The motion is estimated (ME) by variable size block matching with the FIBME algorithm (fast in-band motion estimation) described in Section 2.1. Finally, wavelet coefficients are entropy coded with EZBC (embedded zero-block coding) while motion vectors are encoded in a scalable way by the algorithm proposed in Section 2.3.

In the following, we concentrate on the description of the in-band MCTF module, as we need the background for introducing the proposed fast motion estimation algorithm. MCTF is usually performed taking advantage of the lifting implementation. This technique enables to split direct wavelet temporal filtering into a sequence of prediction and update steps in such a way that the process is both perfectly invertible and computationally efficient. In our implementation a simple Haar transform is used, although the extension to longer filters such as $5 / 3[6,7]$ is conceptually straightforward. In the Haar case, the input frames are recursively processed two-by-two, according to the following equations:

$$
\begin{aligned}
H & =\frac{1}{\sqrt{2}}\left[B-W_{A \rightarrow B}(A)\right], \\
L & =\sqrt{2} A+W_{B \rightarrow A}(H),
\end{aligned}
$$

where $A$ and $B$ are two successive frames and $W_{B \rightarrow A}(\cdot)$ is a motion warping operators that warps frame $A$ into the coordinate system of frame $B . L$ and $H$ are, respectively, the low-pass and high-pass temporal subbands. These two lifting steps are then iterated on the $L$ subbands of the GOP such that for each GOP only one low-pass subband is obtained.

The prediction step is the counterpart of motion compensated prediction in conventional closed loop schemes. The energy of frame $H$ is lower than that of the original frame, thus achieving compression. On the other hand, the update step can be thought as a motion-compensated averaging along the motion trajectories: the updated frames are free from temporal aliasing artifacts and at the same time $L$ requires fewer bits for the same quality than frame $A$ because of the motion-compensated denoising performed by the update step.

In the $2 \mathrm{D}+\mathrm{t}$ scenario, temporal filtering occurs in the wavelet domain and the reference frame is thus available in 


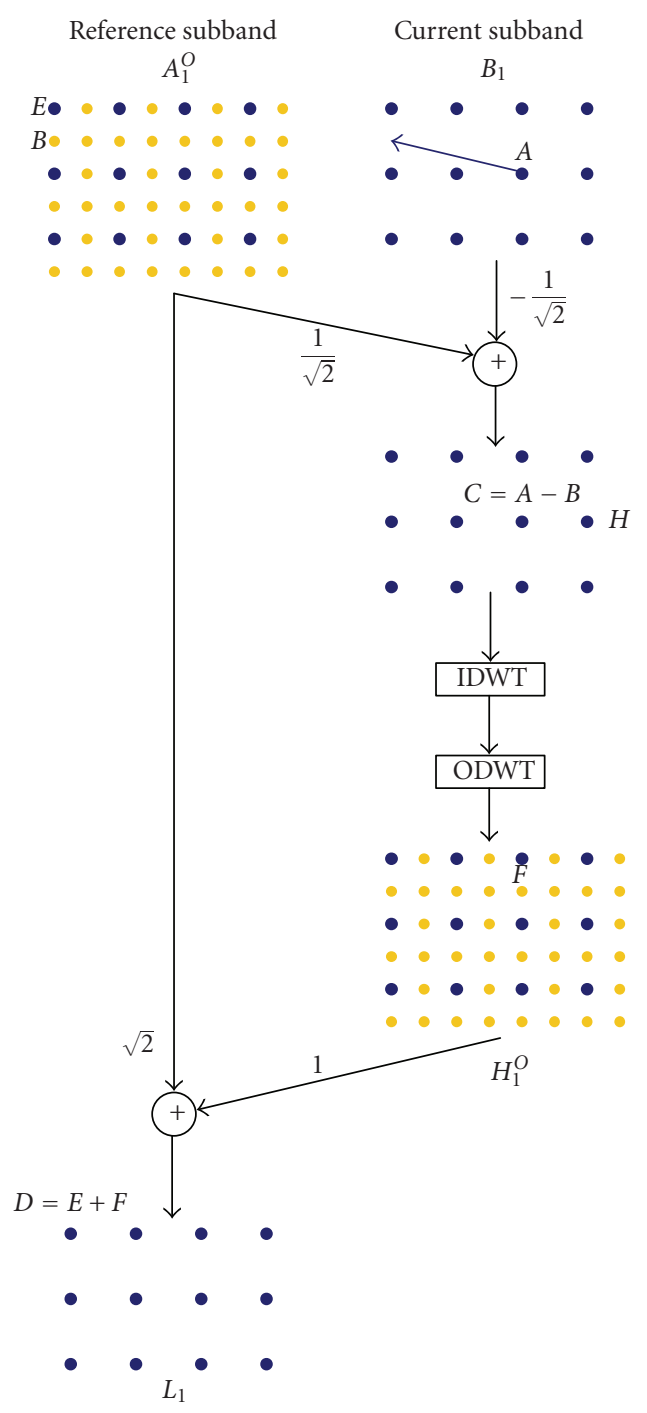

(a)

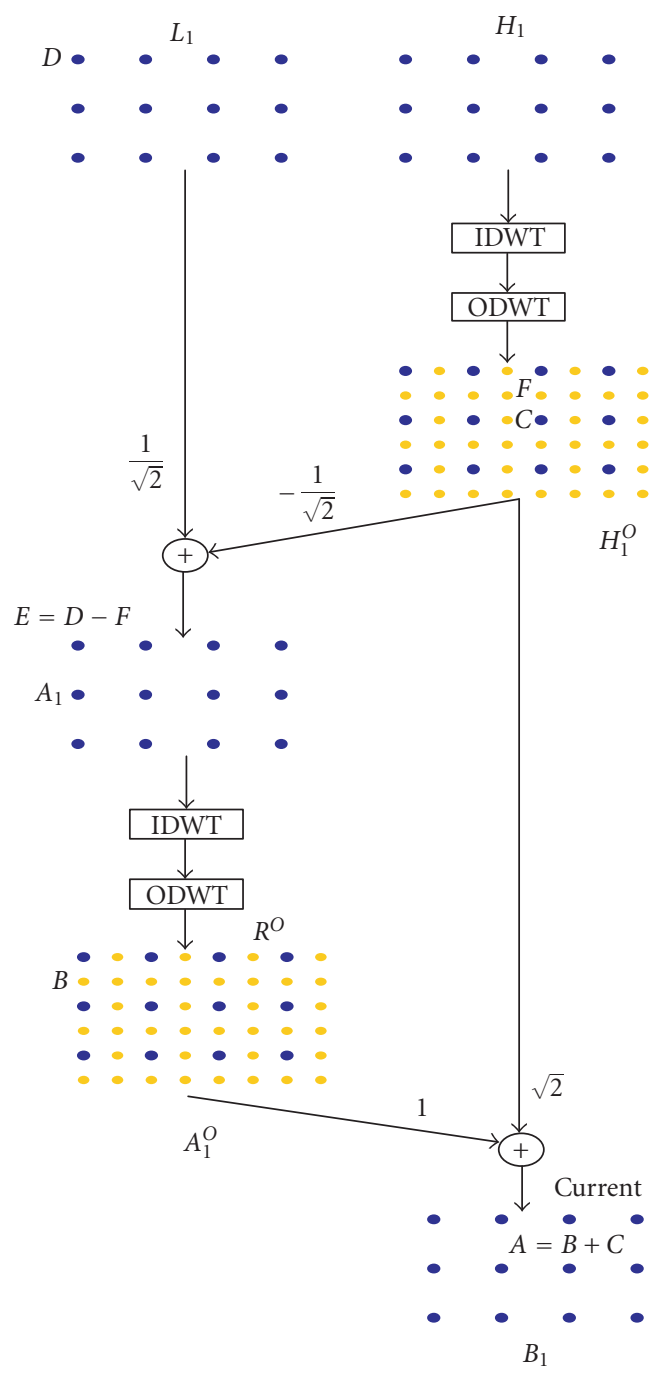

(b)

FIGURE 3: In-band MCTF: (a) temporal filtering at the encoder side (MCTF analysis); (b) temporal filtering at the decoder side (MCTF synthesis).

its overcomplete version in order to combat the shift variance of the wavelet transform. In what follows we illustrate an implementation of the lifting structure, which works in the overcomplete wavelet domain. Figure 3 shows the current and the overcomplete reference frame together with the estimated motion vector $(d x, d y)$ in the wavelet domain. For the sake of clarity, we refer to one wavelet subband at decomposition level $1\left(L H_{1}, H L_{1}\right.$, or $\left.H H_{1}\right)$. The computation of $H_{i}$ is rather straightforward. For each coefficient of the current frame, the corresponding wavelet transformed coefficient in the overcomplete transformed reference frame is subtracted:

$$
H_{i}(x, y)=\frac{1}{\sqrt{2}}\left[B_{i}(x, y)-A_{i}^{O}\left(2^{i} x+d x, 2^{i} y+d y\right)\right],
$$

where $A_{i}^{O}$ is the overcomplete wavelet-transformed reference frame subband at level $i$ and it has the same number of samples as the original frame. The computation of the $L_{i}$ subband is not as trivial. While $H_{i}$ shares the coordinate system with the current frame, $L_{i}$ uses the reference frame coordinate system. A straightforward implementation could be

$$
L_{i}(x, y)=\sqrt{2} A_{i}^{O}\left(2^{i} x, 2^{i} y\right)+H_{i}\left(x-d x / 2^{i}, y-d y / 2^{i}\right) .
$$

The problem here is that the coordinates $\left(x-d x / 2^{i}, y-d y / 2^{i}\right)$ might be noninteger valued even if full pixel accuracy of the displacements is used in the spatial domain. Consider, for example, the coefficient $D$ in the $L_{i}$ subband, as shown in Figure 3. This coefficient should be computed as the sum between coefficients $E$ and $F$. Unfortunately, the latter does not exist in subband $H_{i}$, which suggests that an interpolated version of $H_{i}$ is needed. First, we need to compute the inverse DWT (IDWT) of the $H_{i}$ subbands, which transforms it back to the spatial domain. Then, we obtain the overcomplete 
DWT, $H_{i}^{O}$. The $L_{i}$ frame can be now computed as

$$
\begin{aligned}
L_{i}(x, y)= & \sqrt{2} A_{i}^{O}\left(2^{i} x, 2^{i} y\right) \\
& +\operatorname{ODWT}\left(\operatorname{IDWT}\left(H_{i}\right)\right)\left(2^{i} x-d x, 2^{i} y-d y\right) \\
= & \sqrt{2} A_{i}^{O}\left(2^{i} x, 2^{i} y\right)+H_{i}^{O}\left(2^{i} x-d x, 2^{i} y-d y\right) .
\end{aligned}
$$

The process is repeated in reverse order at the decoder side. The decoder receives $L_{i}$ and $H_{i}$. First the overcomplete copy of $H_{i}$ is computed through IDWT-ODWT. The reference frame is reconstructed as

$$
\begin{aligned}
A_{i}(x, y)=\frac{1}{\sqrt{2}}[ & L_{i}(x, y)-\operatorname{ODWT}\left(\operatorname{IDWT}\left(H_{i}\right)\right) \\
& \left.\times\left(2^{i} x-d x, 2^{i} y-d y\right)\right] \\
= & \frac{1}{\sqrt{2}}\left[L_{i}(x, y)-H_{i}^{O}\left(2^{i} x-d x, 2^{i} y-d y\right)\right] .
\end{aligned}
$$

At this point, the overcomplete version of the reference frame must be reconstructed via IDWT-ODWT in order to compute the current frame:

$$
\begin{aligned}
B_{i}(x, y)= & \sqrt{2} H_{i}(x, y) \\
& +\operatorname{ODWT}\left(\operatorname{IDWT}\left(A_{i}\right)\right)\left(2^{i} x-d x, 2^{i} y-d y\right) \\
= & \sqrt{2} H_{i}^{O}\left(2^{i} x-d x, 2^{i} y-d y\right) \\
& +A_{i}^{O}\left(2^{i} x+d x, 2^{i} y+d y\right) .
\end{aligned}
$$

Figure 3 shows the overall process diagram that illustrates the temporal analysis at the encoder and the synthesis at the decoder. Notice that the combined IDWT-ODWT operation takes place three times, once at the encoder and twice at the decoder. In the actual implementation, the IDWTODWT cascade can be combined in order to reduce the memory bandwidth and the computational complexity according to the complete-to-overcomplete (CODWT) algorithm described in [20].

\subsection{Fast in-band motion estimation}

The wavelet in-band prediction mechanism $(2 \mathrm{D}+\mathrm{t})$, as illustrated in [9], works by computing the residual error after block matching. For each wavelet block, the bestmatching wavelet block is searched in the overcomplete wavelet-transformed reference frame, using a full search approach. The computational complexity can be expressed in terms of the number of required operations as

$$
T=2 W^{2} N^{2},
$$

where $W$ is the size of the search window and $N$ is the block size. As a matter of fact, for every motion vector, at least $N^{2}$ subtractions and $N^{2}$ summations are needed to compute the MAD (mean absolute difference) of the residuals, and there exist $W^{2}$ different motion vectors to be tested.

The proposed fast motion estimation algorithm is based on optical flow estimation techniques. The family of differential algorithms, including Lucas-Kanade [30] and HornSchunk [31], assumes that the intensity remains unchanged along the motion trajectories. This results in the brightness constraint in differential form:

$$
I_{x} v_{x}+I_{y} v_{y}+I_{t}=0
$$

where,

$$
I_{x}=\frac{\partial I(x, y, t)}{\partial x}, \quad I_{y}=\frac{\partial I(x, y, t)}{\partial y}, \quad I_{x}=\frac{\partial I(x, y, t)}{\partial t},
$$

$I_{x}, I_{y}$, and $I_{t}$ are the horizontal, vertical, and temporal gradients, respectively. Notice that when $I_{x} \gg I_{y}$, that is, when the local texturing is almost vertically oriented, only the $d x$ $\left(d x=v_{x} d t\right)$ component can be accurately estimated because:

$$
\frac{I_{x}}{I_{x}} v_{x}+\frac{I_{y}}{I_{x}} v_{y}+\frac{I_{t}}{I_{x}} \simeq v_{x}+\frac{I_{t}}{I_{x}}=0 .
$$

This is the so-called "aperture problem" [32], which consists of the fact that when the observation window is too small, we can only estimate the optical flow component that is parallel to the local gradient. That of the aperture is indeed a problem for traditional motion estimation methods, but in the proposed motion estimation algorithm we take advantage of this fact.

For the sake of clarity, let us consider a pair of images that exhibit a limited displacement between corresponding elements, and let us focus on the $H L$ subband only (before subsampling). This subband is low-pass filtered along the vertical axis and high-pass filtered along the horizontal axis. The output of this separable filter looks like the spatial horizontal gradient $I_{x}$. In fact, the $H L$ subbands tend to preserve only those details that are oriented along the vertical direction. This suggests us that the family of $H L$ subbands, all sharing the same orientation, could be used to accurately estimate the $d x$ motion vector component. Similarly, $L H$ subbands have details oriented along the horizontal axis, therefore they are suitable for computing the $d y$ component. For each wavelet block, a coarse full search is applied to the $L L_{K}$ subband only, where the subscript $K$ is the number of the considered DWT decomposition levels. This initial computation allows us to determine a good starting point $\left(d x^{\mathrm{FS}}, d y^{\mathrm{FS}}\right)^{1}$ for the fast search algorithm, which reduces the risk of getting trapped into local minima. As the $L L_{K}$ subband has $2^{2 K}$ fewer samples than the whole wavelet block, block matching is not computationally expensive. In fact, the computational complexity of this initial step expressed in terms of the number of additions and multiplications is

$$
T=2\left(\frac{W}{2^{K}}\right)^{2}\left(\frac{N}{2^{K}}\right)^{2}=\frac{W^{2} N^{2}}{2^{4 K-1}}
$$

At this point we can focus on the $H L$ subbands. In fact, we use a block matching process on these subbands in order to compute the horizontal displacements and estimate the $d x$

\footnotetext{
1 The superscript FS stands for full search.
} 
component for block $k$ whose top-left corner has coordinates $\left(x_{k}, y_{k}\right)$. The search window is reduced to $W / 4$, as we only need to refine the coarse estimate provided by the full search:

$$
\begin{aligned}
d x_{k}=\arg \min _{d x_{j}} \sum_{i=1}^{K} & \operatorname{MAD}_{H L_{i}}\left(x_{k}, y_{k}, d x^{\mathrm{FS}}+d x_{j}, d y^{\mathrm{FS}}\right) \\
& +\operatorname{MAD}_{L L_{K}}\left(x_{k}, y_{k}, d x^{\mathrm{FS}}+d x_{j}, d y^{\mathrm{FS}}\right),
\end{aligned}
$$

where $\operatorname{MAD}_{H L_{i}}\left(x_{k}, y_{k}, d x, d y\right)$ and $\operatorname{MAD}_{L L_{K}}\left(x_{k}, y_{k}, d x, d y\right)$ are the MAD obtained compensating the block $\left(x_{k}, y_{k}\right)$ in the subbands $H L_{i}$ and $L L_{K}$, respectively, with the motion vector $(d x, d y)$ :

$$
\begin{aligned}
& \operatorname{MAD}_{H L_{i}}\left(x_{k}, y_{k}, d x, d y\right) \\
& =\sum_{x=x_{k, i}}^{x_{k, i}+N / 2^{i}} \sum_{y=y_{k, i}}^{y_{k, i}+N / 2^{i}}\left|H L_{i}^{\mathrm{cur}}(x, y) H L_{i}^{\mathrm{ref}^{O}}\left(2^{i} x+d x, 2^{i} y+d y\right)\right|, \\
& \operatorname{MAD}_{L L_{K}}\left(x_{k}, y_{k}, d x, d y\right) \\
& =\sum_{x=x_{k, i}} \sum_{y=y_{k, i}}^{x_{k, i}+N / 2^{K}}\left|L L_{K}^{\mathrm{cur}}(x, y) L L_{K}^{\mathrm{ref}_{k, i}^{O}+N / 2^{K}}\left(2^{K} x+d x, 2^{K} y\right)\right|,
\end{aligned}
$$

$\left(x_{k, i}, y_{k, i}\right)$ are the top-left corner coordinates of block $k$ subband at level $i$ and it is equal to $\left(\left\lfloor x_{k} / 2^{i}\right\rfloor,\left\lfloor y_{k} / 2^{i}\right\rfloor\right)$.

Because of the shift-varying behavior of the wavelet transform, block matching is performed considering the overcomplete DWT of the reference frame $\left(H L_{i}^{\text {ref }^{\mathrm{O}}}(\cdot)\right.$ and $\left.L L_{K}^{\text {ref }}{ }^{O}(\cdot)\right)$. Similarly we can work on the $L H$ subbands to estimate the $d y$ component. In order to improve the accuracy of the estimate, this second stage takes $\left(x_{k}+d x^{\mathrm{FS}}+d x_{k}, y_{k}+d x^{\mathrm{FS}}\right)$ as a starting point:

$$
\begin{array}{r}
d y_{k}=\arg \min _{d y_{j}} \sum_{i=1}^{K} \operatorname{MAD}_{L H_{i}}\left(x_{k}, y_{k}, d x^{\mathrm{FS}}+d x_{k}, d y^{\mathrm{FS}}+d y_{j}\right) \\
+\operatorname{MAD}_{L L_{K}}\left(x_{k}, y_{k}, d x^{\mathrm{FS}}+d x_{k}, d y^{\mathrm{FS}}+d y_{j}\right),
\end{array}
$$

where $\operatorname{MAD}_{L H_{i}}\left(x_{k}, y_{k}, d x, d y\right)$ is defined in a similar way to $\operatorname{MAD}_{H L_{i}}\left(x_{k}, y_{k}, d x, d y\right)$.

We refer to this algorithm for motion estimation as fast in-band motion estimation (FIBME). The algorithm achieves a good solution that compares favorably with respect to a full search approach with a modest computational effort. The computational complexity of this method is

$$
T \simeq 2 \cdot \frac{2}{3} \cdot \frac{W}{4} N^{2}+\frac{W^{2} N^{2}}{2^{4 K-1}}=\frac{1}{3} W N^{2}+\frac{W^{2} N^{2}}{2^{4 K-1}},
$$

where $W / 4$ comparisons are required to compute the horizontal component and other $W / 4$ for the vertical component. Each comparison involves either $H L$ or $L H$ subbands, whose size is approximately one third of the whole wavelet block (if we neglect the $L L_{K}$ subband). If we keep the block size
$N$ fixed, the proposed algorithm runs in linear time with the search window size, while the complexity of the full search grows with the square power. The speedup factor with respect to the full search in terms of number of operations is

$$
\text { speedup }=\frac{2 W^{2} N^{2}}{(1 / 3) W N^{2}+W^{2} N^{2} / 2^{4 K-1}} \simeq 6 W .
$$

It is worth pointing out that this speedup factor refers to the motion estimation task, which is only part of the overall computational burden at the encoder. In Section 3, we give more precise indications, based on experimental evidence, about the actual encoding time speedup, including wavelet transforms, motion compensation, and entropy coding. At a fraction of the cost of the full search, the proposed algorithm achieves a solution that is suboptimal. Nevertheless, Section 3 shows through extensive experimental results on different test sequences that the coding efficiency loss is limited approximately to $0.5 \mathrm{~dB}$ on sequences with large motion.

We have investigated the accuracy of our search algorithm in case of large displacements. If we do not use a full search for the $L L_{K}$ subband, our approach tends to give a bad estimate of the horizontal component when the vertical displacement is too large. In this scenario, when the search window scrolls horizontally, it cannot match the reference displaced block. We observed that the maximum allowed vertical displacement is approximately as large as the low-pass filter impulse response used by the critically sampled DWT. This is due to the fact that such filter operates along the vertical direction by stretching the details proportionally to its impulse response extension.

The same conclusions can be drawn if we take a closer look at Figure 4. A wavelet block from the DWT-transformed current frame is taken as the current block, while the ODWT transformed reference frame is taken as the reference. For all possible displacements $(d x, d y)$, the MAD of the prediction residuals is computed by compensating only the $H L$ subband family, that is, the one that we argue being suitable for estimating the horizontal displacement. In Figure 4(a), the global minimum of this function is equal to zero and is located at $(0,0)$. In addition, around the global minimum there is a region that is elongated in the vertical direction, which is characterized by low values of the MAD. Let us now consider a sequence of two images, one obtained from the other through translation of the vector $\left(d x^{\prime}, d y^{\prime}\right)=(10,5)$ (see Figure 4(b)). Considering a wavelet block on the current image, Figure 4 shows the MAD value for all the possible displacements. A full search algorithm would identify the global minimum $M$. Our algorithm starts from point $A(0,0)$ and proceeds horizontally both ways to search for the minimum $(B)$. If $d y^{\prime}$ is not too large, the horizontal search finds its optimum in the elongated valley centered on the global minimum, therefore the horizontal component is estimated quite accurately. The vertical component can now be estimated without problems using the $L H$ family subbands. In conclusion, coarsely initializing the algorithm with a full search provides better results in case of large $d y^{\prime}$ displacement without significantly affecting the computational complexity. 


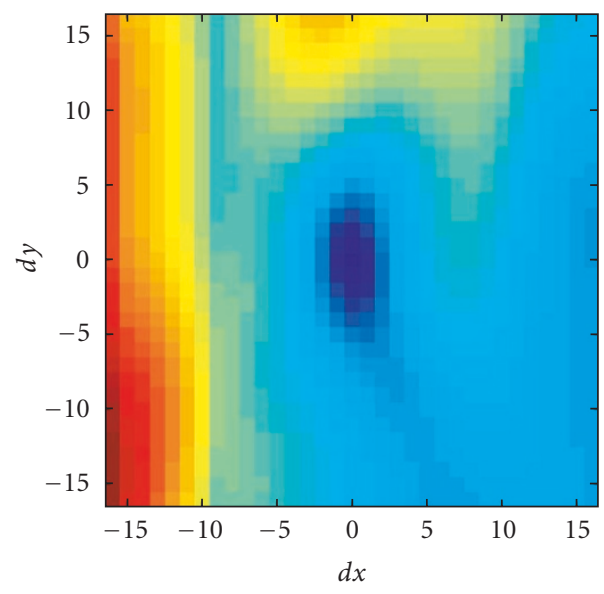

(a)

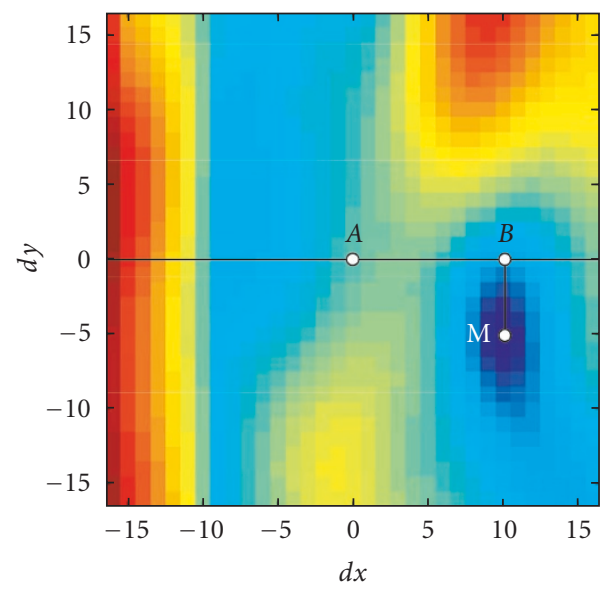

(b)

FIgURE 4: Error surface as a function of the candidate motion vector $(d x, d y)$ : (a) global minimum in $(0,0)$; (b) global minimum in $(15,5)$.

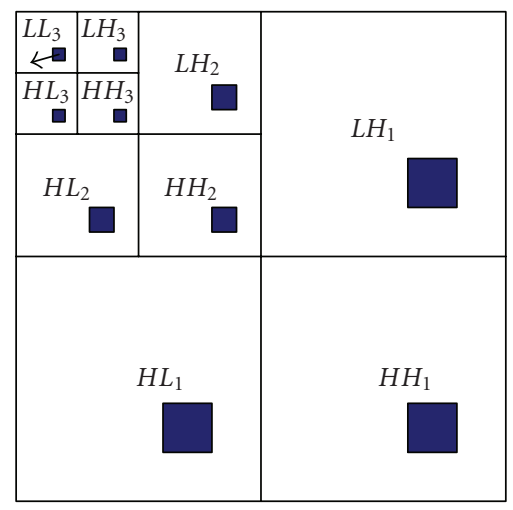

(a)

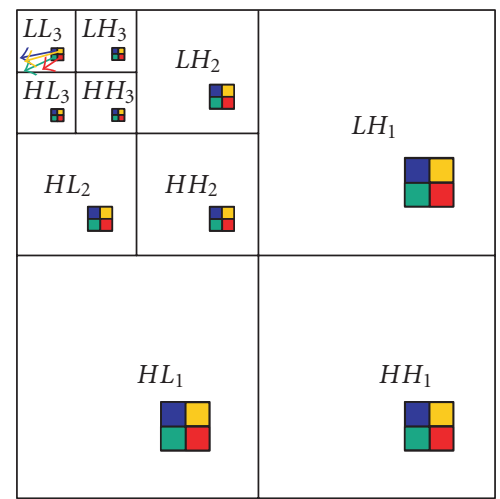

(b)

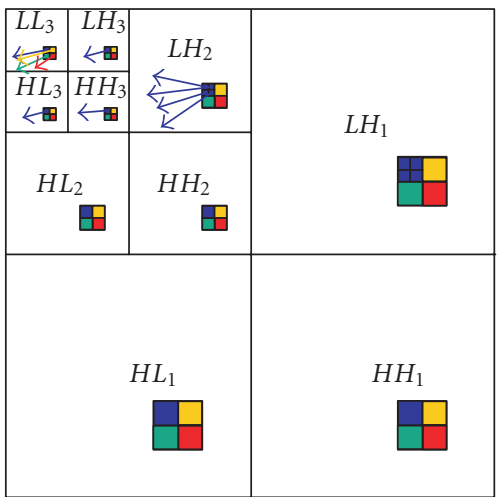

(c)

FIgURe 5: Motion field assigned to a $16 \times 16$ wavelet block: (a) one vector per wavelet block; (b) four vectors per wavelet block; (c) further splitting of the wavelet subblock.

\subsection{Variable size block matching}

As described so far, the FIBME fast search algorithm works with wavelet blocks of fixed sizes. We propose a simple extension that allows to adopt blocks of variable sizes by generalizing the HVSBM (hierarchical variable size block matching) [23] algorithm to work in the wavelet domain. Let us consider a three-level wavelet decomposition and a wavelet block of size $16 \times 16$ (refer to Figure $5(\mathrm{a})$ ). In the fixed size implementation, only one motion vector is assigned to each wavelet block. If we focus on the lowest frequency subband, the wavelet block covers a $2 \times 2$ pixel area. Splitting this area into four and taking the descendants of each element, we generate four $8 \times 8$ wavelet blocks, which are the offspring of the $16 \times 16$ parent block (see Figure 5(b)). Block matching is performed on those smaller wavelet blocks to estimate four distinct motion vectors. In that figure, all the elements that have the same color are assigned the same motion vector.
Like in HVSBM, we build a quadtree-like structure where in each node we store the motion vector, the rate $R$ needed to encode the motion vector and the distortion $D$ (MAD). A pruning algorithm is then used to select the optimal splitting configuration for a given bitrate budget [23]. The number $B$ of different block sizes relative to the wavelet block size $N$ and the wavelet decomposition level $K$ is

$$
B=\log _{2}\left(\frac{N}{2^{K}}\right)+1
$$

that corresponds to the block sizes:

$$
\left(\frac{N}{2^{i}} \times \frac{N}{2^{i}}\right), \quad i=0, \ldots, B-1 .
$$

If we do not want to be forced to work with fixed size blocks, we need to have at least two different block sizes. For 
example, with $B=2$, we have

$$
\log _{2}\left(\frac{N}{2^{K}}\right)>1 \Longrightarrow \frac{N}{2^{K}}>2 \Longrightarrow N>2^{K+1} .
$$

Having fixed $K$, the previous equation sets a lower bound on the size of the smallest wavelet block. By setting $N=16$ and $K=3$, three different block sizes are allowed: $16 \times 16$, $8 \times 8$, and $4 \times 4$. We can take this approach one step further in order to overcome the lower bound. If $N=2^{K}$ or if we have already split the wavelet block in such a way that there is only one pixel in the $L L_{K}$ subband, further split can be performed according to the above scheme. In order to provide a motion field of finer granularity, we can still assign a new motion vector to each subband $L H_{K}, H L_{K}, H H_{K}$, plus the refined version of $L L_{K}$ alone. This way we produce four children motion vectors, as shown in Figure 5(c). In this case, the motion vector shown in subband $\mathrm{HL}_{3}$ is the same one used for compensating all of the coefficients in subbands $H L_{3}, H L_{2}$, and $H L_{1}$. The same figure shows a further splitting step performed on the wavelet block of the $\mathrm{LH}_{3}$ subband. In fact, the splitting can be iterated at lower scales, by assigning one motion vector to each one-pixel subblock at level $K-1$ (in subband $\mathrm{LH}_{2}$ in this example). Figure 5(c) shows that the wavelet block with roots on the blue pixel (in the top-left position) in subband $\mathrm{LH}_{3}$, is split into four subblocks in the $\mathrm{LH}_{2}$ subband. These refinement steps allow us to compensate elements in different subbands with different motion vectors that correspond to the same spatial location. We need to emphasize that this last splitting step makes the difference between spatial domain variable size block matching and the proposed algorithm. In fact, in the latter case it is possible to compensate the same spatial region with separate motion vectors, according to the local texture orientation. Following this simple procedure, we can generate subblocks of arbitrary size in the wavelet domain.

\subsection{Scalable coding of motion vectors}

In both $\mathrm{t}+2 \mathrm{D}$ and $2 \mathrm{D}+\mathrm{t}$, wavelet-based video codec SNR scalability is achieved by truncating the embedded representation of the wavelet coefficients. In this way, only the texture information is scaled, while the motion information is lossless encoded, thus occupying a fixed amount of the bit budget decided at encoding time and unaware of the decoding bitrate. This fact has two major drawbacks. First, the video sequence cannot be encoded at a target bitrate lower than the one necessary to lossless encode the motion vectors. Second, no optimal tradeoff between motion and residuals bit budget can be computed.

Recently, it has been demonstrated [26] that in the case of open-loop wavelet-based video coders it is possible to use a quantized version of the motion field during decoding together with the residual coefficients computed at the encoder with the lossless version of the motion. A scalable representation of the motion is achieved in [26] by coding the motion field as a two-component image using a JPEG2000 scheme. This is possible as long as the motion vectors are disposed on a regular lattice, as it is the case for fixed size block matching or deformable meshes using equally spaced control points.
In this section, we introduce an algorithm able to build a scalable representation of the motion vectors which is specifically designed to work with blocks of variable sizes produced in output by the motion estimation algorithm presented in Sections 2.1 and 2.2.

Block sizes range from $N_{\max } \times N_{\max }$ to $N_{\min } \times N_{\min }$ and they tend to be smaller in regions characterized by complex motion. Neighboring blocks usually manifest a high degree of similarity, therefore a coding algorithm able to reduce their spatial redundancy is needed. In the standard implementation of HVSBM [23], a simple nearest neighbor predictor is used for this purpose. Although it achieves a good lossless coding efficiency, it does not provide a scalable representation. The proposed algorithm aims at achieving the same performance when working in lossless mode allowing at the same time a scalable representation of the motion information.

In order to tackle spatial redundancy, a multi-resolution pyramid of the motion field is built in a bottom-up fashion. As shown in Figure 6, variable size block matching generates a quadtree-like representation of the motion model. At the beginning of the algorithm, only the leaf nodes are assigned with a value, representing the two components of the motion vector. For each component, we compute the value of the node as a simple average of its four offspring. Then we code each offspring as the difference between each value and its parent. We iterate these steps further up the motion vector tree. The root node contains an average of the motion vectors over the whole image. Depending on the size of the image and $N_{\min }$, the root node might have fewer than four offspring.

Figure 6 illustrates a toy example that clarifies this multiresolution representation. The motion vector components are the numbers indicated just below each leaf node. The averages computed on intermediate nodes are shown in grey, while the values to be encoded are written in bold typeface. The same figure also shows the labeling convention we use: each node is identified by a pair $(i, d)$, where $d$ represents the depth in the tree while $i$ is the index number starting from zero of the nodes at a given depth. Since the motion field usually exhibits a certain amount of spatial redundancy, the leaf nodes are likely to have a smaller absolute values. In other words, walking down from the root to the leaves, we can expect the same sort of energy decay that is specific of wavelet coefficients across subbands following parent-children relationships. This fact suggested us that the same ideas underpinning wavelet-based image coders could be exploited here. Specifically, if an intermediate node is insignificant with respect with a given threshold, then it is likely that its descendants are also insignificant. This is the reason why the proposed algorithm inherits some of the basic concepts of SPIHT [2] (set partitioning in hierarchical trees).

Before detailing the steps of the algorithm, it is important to point out that, in the quadtree representation that we have built so far, the node values should be multiplied by a weighting factor that depends on their depth in the tree. Let us consider only one node and its four offspring. If we wish to achieve a lossy representation of the motion field, these nodes 


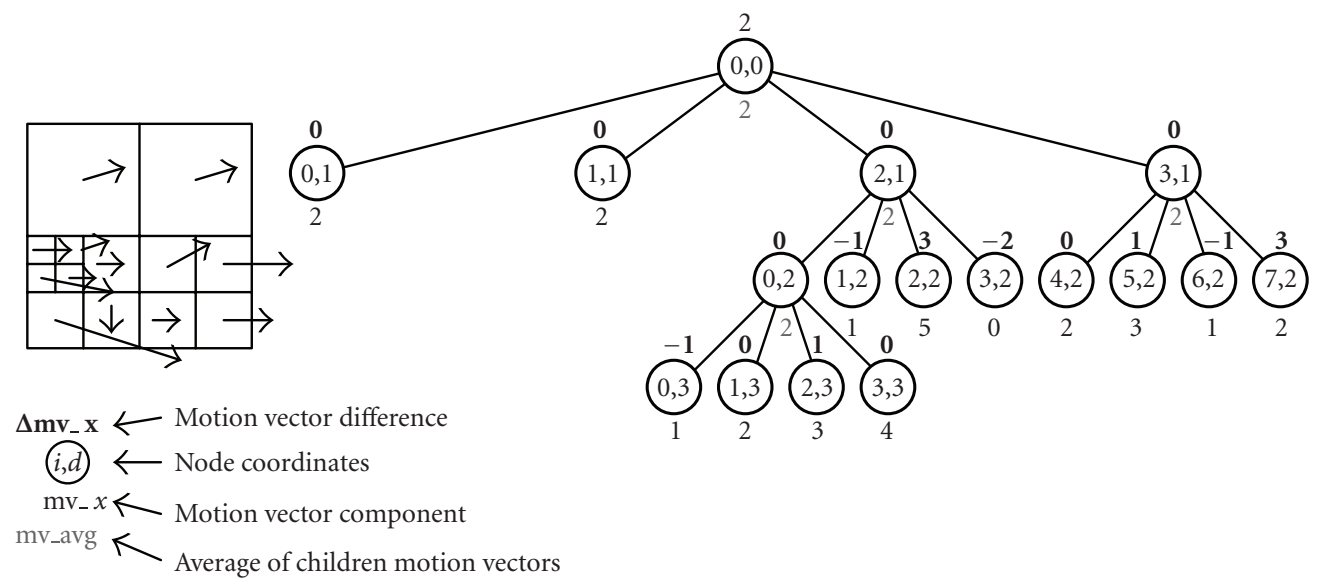

FIGURE 6: Quadtree-like representation of the motion model generated by the variable size block matching algorithm.

will be quantized. If we make an error in the parent node, that will badly affect its offspring, while the same error will have fewer consequences if one of the children is involved. If we use the mean squared error as a distortion measure, the parent node needs to be multiplied by a factor of 2 , in such a way that errors are weighted equally and the same quantization step sizes can be used regardless of the node depth.

The proposed algorithm encodes the nodes of the quadtree from top to bottom starting from the most significant bitplane. As in SPIHT, the algorithm is divided into a sorting pass that identifies which nodes are significant with respect to a given threshold, and a refinement pass that refines the nodes already found significant in the previous steps. There are four lists that are maintained both at the encoder and the decoder, which allow to keep track of each node status. The LIV (list insignificant vectors) contains those nodes that have not been found significant yet. The LIS (list insignificant sets) represents those nodes whose descendants are insignificant. On the other hand, LSV (list significant vectors) and LSS (list significant sets) contain either nodes found significant or whose descendants are significant. A node can be moved from LIV to LIS and from LIS to LSS, but not vice versa. Only the nodes in the LSV are refined during the refinement pass. The following notation is used:

(i) $P(i, d)$ : coordinates of parent node of node $i$ at depth $d$;

(ii) $O(i, d)$ : set of coordinates of all offspring of node $i$ at depth $d$;

(iii) $D(i, d)$ : set of coordinates of all descendants of node $i$; at depth $d$;

(iv) $H(0,0)$ : coordinate of the quadtree root node.

The algorithm is described in detail by pseudocode listed in Algorithm 1. Note that $\hat{d}$ keeps track of the depth of the current node. This way instead of scaling by a factor of 2 all the intermediate nodes with respect to their offspring, the significance test is carried out at bitplane $n+\hat{d}$, that is, $S_{n+\hat{d}}\left(i_{\hat{d}}, \hat{d}\right)$.

As for SPIHT, encoding and decoding use the same algorithm, where the word output is substituted by input at the decoder side. The symbols emitted by the encoder are arithmetic coded.

The bitstream produced by the proposed algorithm is completely embedded, in such a way that it is possible to truncate it at any point and obtain a quantized representation of the motion field. In [26], it is proved that for small displacement errors, there is a linear relation between the MSE (mean square error) of the quantized motion field parameters $\left(\mathrm{MSE}_{W}\right)$ and the MSE of the prediction residue $\left(\mathrm{MSE}_{r}\right)$ :

$$
\mathrm{MSE}_{r}=k \frac{\psi_{x}+\psi_{y}}{2} \mathrm{MSE}_{W}
$$

where the motion sensitivity factors are defined as

$$
\begin{aligned}
& \psi_{x}=\frac{1}{(2 \pi)^{2}} \iint S_{f}(\underline{\omega}) \omega_{x}^{2} d \omega, \\
& \psi_{y}=\frac{1}{(2 \pi)^{2}} \iint S_{f}(\underline{\omega}) \omega_{y}^{2} d \omega,
\end{aligned}
$$

where $S_{f}(\underline{\omega})$ is the power spectrum of the current frame $f(x, y)$. Using this result it is possible to estimate a priori the optimal bit allocation between motion information and residual coefficients [26]. Informally speaking, at low bitrates the motion field can be heavily quantized in order to reduce its bit budget and save bits to encode residual information. On the other hand, at high bitrates the motion field is usually sent lossless as it occupies a small fraction of the overall target bitrate.

\subsection{Motion vectors and spatial scalability}

A spatially scalable video codec is able to deliver a sequence at a lower resolution than the original one in order to fit the receiving device display capabilities. Wavelet-based video coders address spatial scalability in a straightforward way. At the end of spatio-temporal analysis each frame of a GOP of size $T$ represents a temporal subband further decomposed into spatial subbands up to level $K$. Each GOP thus consists 
(1) Initialization:

(1.1) output $\mathrm{msb}=n=\left\lfloor\log _{2} \max _{(i, d)}\left(c_{i, d}\right)\right\rfloor$

(1.2) output max_depth $=\max (d)$

(1.3) set the LLS and the LSV as empty lists add $H(0,0)$ to the LIV and to the LIS.

(2) Sorting pass

(2.1) set $\hat{d}=0$, set $i_{d}=0(d=0,1, \ldots$, max _depth $)$

(2.2) if $0 \leq n+\hat{d} \leq \mathrm{msb}$ do:

(2.2.1) if entry $\left(i_{\hat{d}}, \hat{d}\right)$ is in the LIV do:

(i) output $S_{n+\hat{d}}\left(i_{\hat{d}}, \hat{d}\right)$

(ii) if $S_{n+\hat{d}}\left(i_{\hat{d}}, \hat{d}\right)=1$ then move $\left(i_{\hat{d}}, \hat{d}\right)$ to LSV and output the sign of $c_{i_{\hat{d}}, \hat{d}}$

(2.3) if entry $\left(i_{\hat{d}}, \hat{d}\right)$ is in the LIS do:

(2.3.1) if $n+\hat{d}<\mathrm{msb}$ do:

(i) $S_{D}=0$

(ii) for $h=\hat{d}+1$ to max_depth do:

- for each $(j, h) \in D\left(i_{\hat{d}}, \hat{d}\right)$, if $S_{n+h}(j, h)=1$ then $S_{D}=1$

(iii) output $S_{D}$

(iv) if $S_{D}=1$ then move $\left(i_{\hat{d}}, \hat{d}\right)$ to LSS, add each $(k, l) \in O\left(i_{\hat{d}}, \hat{d}\right)$ to the LIV and to the LIS, increment $\hat{d}$ by 1 , and go to Step (2.2)

(2.4) if entry $\left(i_{\hat{d}}, \hat{d}\right)$ is in the LSS the increment $\hat{d}$ by 1 and go to Step (2.2).

(3) Refinement pass

(3.1) if $0 \leq n+\hat{d} \leq \mathrm{msb}$ do:

(i) if entry $\left(i_{\hat{d}}, \hat{d}\right)$ is in the LSV and was not included during the last sorting pass, then output the $n$th most significant bit of $\left|c_{i, \hat{d}}\right|$

(3.2) if $\hat{d} \geq 1$ do

(i) increment $i_{\hat{d}}$ by 1

(ii) if $\left(i_{\hat{d}}, \hat{d}\right) \in O\left(P\left(i_{\hat{d}-1, \hat{d}}\right)\right)$ then go to Step (2.2); otherwise decrement $\hat{d}$ by 1 and go to Step (3).

(4) Quantization step update: decrement $n$ by 1 and go to Step (2).

Algorithm 1: Pseudocode of the proposed scalable motion vector encoding algorithm.

of the following subbands: $L L_{i}^{t}, L H_{i}^{t}, H L_{i}^{t}, H H_{i}^{t}$ with spatial subband index $i=1, \ldots, K$ and temporal subband index $t=$ $1, \ldots, T$. Let us assume that we want to decode a sequence at a resolution $2^{(k-1)}$ times lower than the original one. We need to send only those subbands with $i=k, \ldots, K$. At the decoder side, spatial decomposition and motion-compensated temporal filtering is inverted in the synthesis phase. It is a decoder task to adapt the full resolution motion field to match the resolution of the received subbands.

In this section we compare analytically the following two approaches:

(a) the original motion vectors are truncated and rounded in order to match the resolution of the decoded sequence,

(b) the original motion vectors are retained, while a full resolution sequence is interpolated starting from the received subbands.
The former implementation tends to be computationally simpler while not as efficient as the latter in terms of coding efficiency as it will be demonstrated in the following. Furthermore, this is the technique adopted in the MC-EZBC [5] reference software, used as a benchmark in Section 3.

Let us concentrate our attention on a one-dimensional discrete signal $x(n)$ and its translated version by an integer displacement $d$, that is, $y(n)=x(n-d)$. Their 2D counterpart are the current and the reference frame, respectively. We are thus neglecting motion compensation errors due to complex motion, reflections, and illumination changes. Temporal analysis is carried out with the lifting implementation of the Haar transform along the motion trajectory $d$ :

$$
\begin{aligned}
& H(n)=\frac{1}{\sqrt{2}}[y(n)-x(n-d)]=0, \\
& L(n)=\sqrt{2} x(n)+H(n+d)=\sqrt{2} x(n),
\end{aligned}
$$


$L(n)$ and $H(n)$ are wavelet-transformed and, in the case of spatial scalability, only a subset of their subbands is sent. If we scale at half the original resolution, the decoder receives the following signals:

$$
\begin{gathered}
H_{\text {low }}(n)=0 \\
L_{\text {low }}(n)=\sqrt{2} x_{\text {low }}(n)=\sqrt{2}[x * h(k)]_{k=2 n} .
\end{gathered}
$$

Temporal synthesis reconstructs a low resolution approximation of the original signals:

$$
\begin{aligned}
& \hat{x}_{\text {low }}(n)=\frac{1}{\sqrt{2}}\left[L_{\text {low }}(n)-H_{\text {low }}\left(n+\frac{d}{2}\right)\right]=x_{\text {low }}(n), \\
& \hat{y}_{\text {low }}(n)=\sqrt{2} H_{\text {low }}(n)+\hat{x}_{\text {low }}\left(n-\frac{d}{2}\right)=x_{\text {low }}\left(n-\frac{d}{2}\right) .
\end{aligned}
$$

We compute the reconstruction error using the spatially lowpass filtered and subsampled version of the original frames as reference:

$$
\begin{gathered}
e_{x}(n)=\hat{x}_{\mathrm{low}}(n)-x_{\mathrm{low}}(n)=0 \\
e_{y}(n)=\hat{y}_{\mathrm{low}}(n)-y_{\mathrm{low}}(n)=x_{\mathrm{low}}\left(n-\frac{d}{2}\right)-y_{\mathrm{low}}(n) .
\end{gathered}
$$

We derive the solution for scenario (b) first, since we will see (a) as a particular case.

First, the decoder reconstructs an interpolated version of the original sequence. This is accomplished by setting to zero the coefficients of the missing subbands before performing the wavelet synthesis. It is worth pointing out that this is equivalent to estimating the missing samples using the wavelet scaling function as interpolating kernel. The reconstruction error can be written as

$$
\sum_{n=0}^{N / 2-1} e_{y}^{2}=\sum_{n=0}^{N-1} e_{\mathrm{rec}}^{2}=\sum_{n=0}^{N / 2-1}\left|x_{\operatorname{rec}_{b}}(n-d)-y_{\mathrm{rec}}(n)\right|^{2} .
$$

The first equivalence holds as far as we use an orthogonal transform to reconstruct a full resolution approximation of the signals.

Figure 7 illustrates how $e_{\text {rec }}(n)$ is computed starting from $x(n)$ and $y(n) . H(z)$ represents the analysis wavelet low-pass filter, while $G(z)$ is the synthesis low-pass filter. In the rest of this paper, we assume that they are Daubechies 9/7 biorthogonal filters. As they are nearly orthogonal, (26) is satisfied in practice. The reconstructed signal $x_{\text {rec }}(n)$ is an approximation of $x(n)$ having the same number of samples. Therefore motion compensation can use the original motion vector $d$. Using the Parseval's theorem and further manipulating the expression, the prediction error in (26) becomes (for any odd displacement $d$ )

$$
\sum_{n=0}^{N / 2-1} e_{\mathrm{rec}_{b}}^{2}=2 \int_{0}^{+\pi}|G(\omega+\pi)|^{2}|H(\omega)|^{2}|X(\omega)|^{2} d \omega .
$$

If $d$ is even the error expression is identically equal to zero. Figure 8 depicts $|G(\omega+\pi)|^{2}$ and $|H(\omega)|^{2}$ together with their

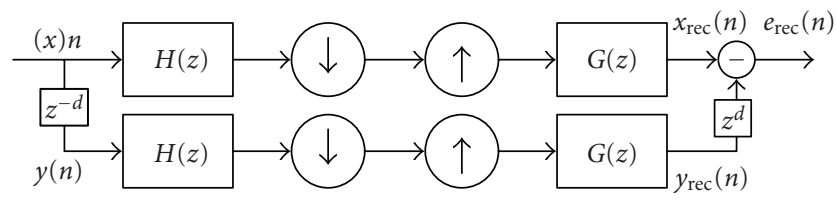

FIGURE 7: Reconstruction error computation without motion vector truncation (scenario (b)).

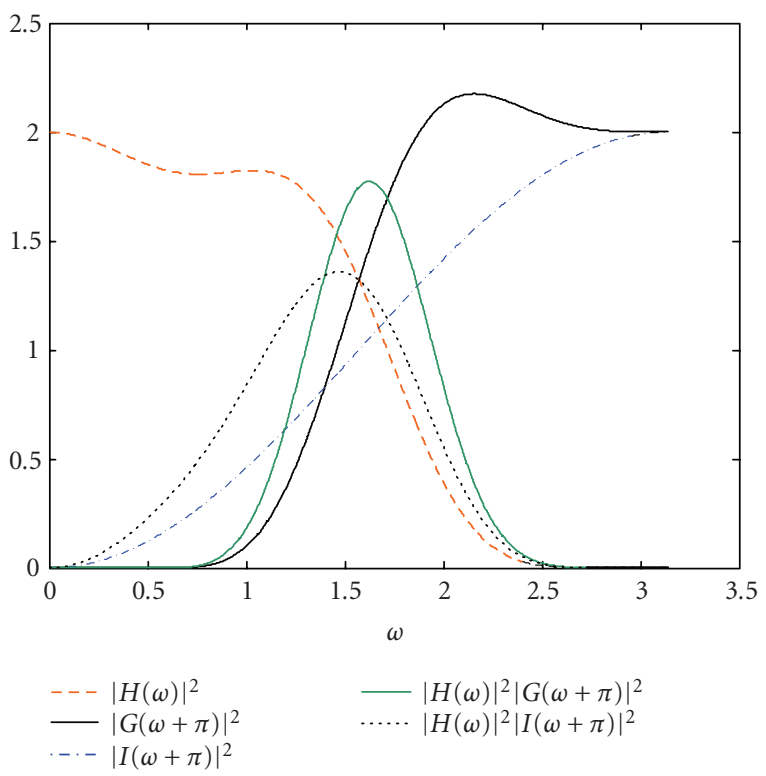

FIGURE 8: Frequency responses of the filters cited in the paper.

product. We can conclude that the error depends on the frequency characteristics of the signal and it is close to zero if its energy is mostly concentrated at low frequencies. Indeed the approximation we get interpolating with $G(\omega)$ is very much similar to the original.

The error in scenario (a) can be derived as a special case of scenario (b). Since the received signal has lower resolution than the motion field, vectors are truncated. If the components are odd, they are also rounded to the nearest integer. The reconstruction error is

$$
\sum_{n=0}^{N / 2-1} e_{y}^{2}=\sum_{n=0}^{N / 2-1}\left|x_{\text {low }}\left(n-\operatorname{round}\left[\frac{d}{2}\right]\right)-y_{\text {low }}(n)\right|^{2}
$$

In order to find a frequency domain expression for this scenario, we can observe that the operation of truncating and rounding motion vectors is equivalent to interpolating the low resolution version received by the decoder with a sample and hold filter and then applying the full resolution motion 


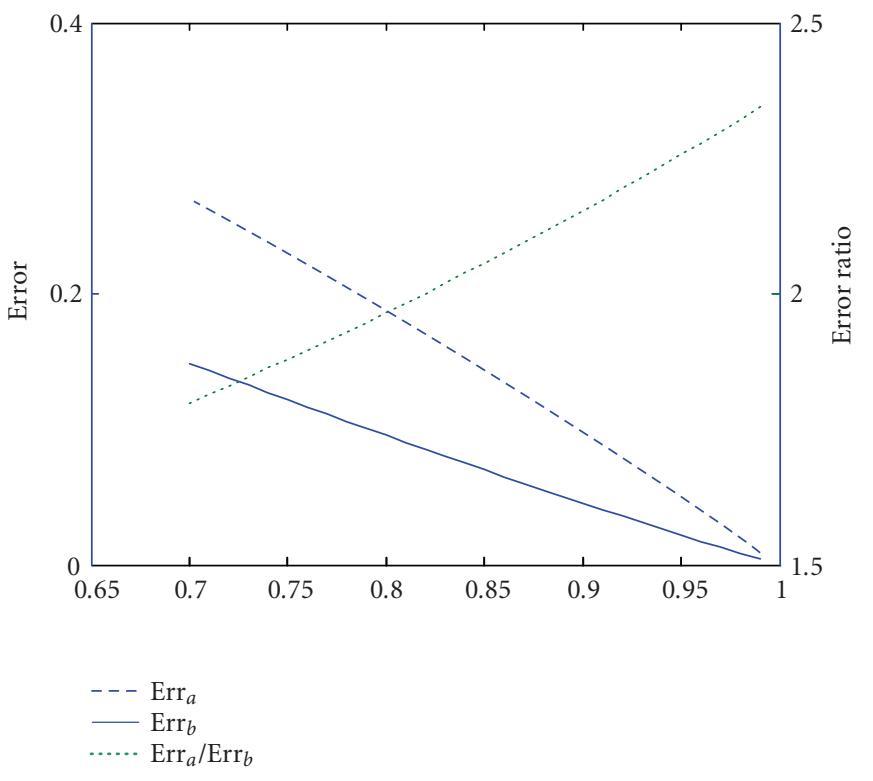

FIGURE 9: Comparison between the (normalized) error in scenario (a) and (b) as a function of the correlation coefficient $\rho$.

field. As a matter of fact, the error can be expressed as

$$
\begin{aligned}
\sum_{n=0}^{N / 2-1} e_{y}^{2} & =\sum_{n=0}^{N-1} e_{\mathrm{rec}_{a}}^{2}(n) \\
& =2 \int_{0}^{+\pi}\left|\frac{e^{j \omega}}{\sqrt{2}}-\frac{1}{\sqrt{2}}\right|^{2}|H(\omega)|^{2}|X(\omega)|^{2} d \omega \\
& =2 \int_{0}^{+\pi}|I(\omega+\pi)|^{2}|H(\omega)|^{2}|X(\omega)|^{2} d \omega .
\end{aligned}
$$

The equivalence holds because the interpolating sample and hold filter $I(\omega)$ is equivalent to the inverse Haar DWT, where the low-frequency subband is the subsampled signal and the high-frequency subband coefficients are set to zero. Having fixed $|H(\omega)|^{2}$, we are unable to state which of the two approaches is better, that is, has smaller error, since $|I(\omega)|^{2}$ is not greater than $|G(\omega)|^{2}$ for all $\omega$ (see Figure 2) and we do not know the power spectrum of the signal. Nevertheless, if we assume that most of the energy is concentrated at low frequencies, approach (b) gives better coding efficiency. In fact, taking the expectation of (27) and (29) with respect to $x(n)$ :

$$
\begin{aligned}
\operatorname{Err}_{a} & =E\left[\sum_{n=0}^{N-1} e_{\operatorname{rec}_{a}}^{2}(n)\right] \\
& =2 \int_{0}^{+\pi}|I(\omega+\pi)|^{2}|H(\omega)|^{2} S_{x}(\omega) d \omega, \\
\operatorname{Err}_{b} & =E\left[\sum_{n=0}^{N-1} e_{\operatorname{rec}_{b}}^{2}(n)\right] \\
& =2 \int_{0}^{+\pi}|G(\omega+\pi)|^{2}|H(\omega)|^{2} S_{x}(\omega) d \omega .
\end{aligned}
$$

If we model the signal as an autoregressive process of order 1 with correlation coefficient $\rho$, the signal power spectrum
$S_{x}(\omega)$ can be expressed in closed form as

$$
S_{x}(\omega)=\frac{1-\rho^{2}}{\left|1-\rho e^{j \omega}\right|^{2}} .
$$

We are now able to evaluate numerically (30). As illustrated in Figure 9, for any $\rho$, in $[0.7,1]^{2} \operatorname{Err}_{a}>\operatorname{Err}_{b}$ and their ratio is higher for $\rho$ close to 1 , meaning that the penalty due to motion vector truncation with respect to interpolating at full resolution with $G(\omega)$ is greater when the input signal has energy concentrated in the low-frequency range.

Section 3 validates the results of the formal analysis by giving experimental evidence about the better coding efficiency achievable without truncating the motion vectors.

\section{EXPERIMENTAL RESULTS}

In this section, we discuss the overall performance of the $2 \mathrm{D}+\mathrm{t}$ scalable video codec presented in Section 2 in terms of complexity and coding efficiency. We emphasize the impact of each of the coding tools detailed in this paper, in order to evaluate the best combination of the coding parameters.

As a benchmark, we use the MC-EZBC codec (motioncompensated embedded zero-block coding) [5] for the $t+2 \mathrm{D}$ case, as the proposed $2 \mathrm{D}+\mathrm{t}$ codec shares some of its functional modules (i.e., spatial and temporal filters, entropy coding algorithm).

Throughout our simulations, we used the following set of parameters:

\footnotetext{
${ }^{2}$ Although Figure 9 is zooming on the $[0.7,1]$ range, the same behavior is
} observed in the whole $[0,1]$ interval. 
(i) sequence spatio-temporal resolution:

- QCIF $(176 \times 144)$ at $30 \mathrm{fps}$ : Silent

- CIF $(352 \times 288)$ at 30 fps: Mobile \& Calendar, Foreman, Football

- 4CIF $(704 \times 576)$ at $60 \mathrm{fps}$ : City, Soccer;

(ii) number of frames: 300;

(iii) spatial wavelet transform: Daubechies 9/7:

- QCIF: $K=3$

- CIF: $K=3$

- 4CIF: $K=4$;

(iv) temporal transform: Haar;

(v) GOP size: 16 frames;

(vi) search window: $[-W / 2,+W / 2]$ :

- QCIF: $[-16,+16]$

- CIF: $[-32,+32]$

- 4CIF: [-48, +48];

(vii) motion accuracy: $1 / 4$ pixel;

(viii) block size:

- QCIF and CIF: fixed size $16 \times 16$, variable size $64 \times 64$ to $4 \times 4$

- 4CIF: fixed size $32 \times 32$, variable size $128 \times 128$ to $4 \times 4$;

(ix) entropy coding: EZBC.

In the first experiment, we compare the proposed fast motion estimation algorithm (FIBME) (see Section 2.1) with the computationally demanding full search strategy. As a reference, we include the rate-distortion curve obtained with MC-EZBC. In terms of encoding complexity, the proposed algorithm allows a speedup factor of the overall encoding phase equal to 18-19. To this regard, Table 1 shows, for each sequence, the encoding time normalized with respect to the encoding time needed when FIBME is used for the same sequence. Note that also MC-EZBC uses a fast motion estimation algorithm (HVSBM [23]).

Figure 10 and Table 2 show the rate-distortion performance of the proposed $2 \mathrm{D}+\mathrm{t}$ codec with FIBME with respect to a full search motion estimation algorithm. In both cases, blocks of variable sizes are used. From these results we can conclude that, apart from the Mobile \& Calendar sequence and Foreman at high bitrates, the rate-distortion gap between FIBME and full search remains within the $0 \mathrm{~dB}-0.3 \mathrm{~dB}$ range. In all of the remaining experiments, the $2 \mathrm{D}+\mathrm{t}$ codec always uses the FIBME algorithm in the motion estimation phase.

Figure 11 and Table 3 show the objective PSNR gain that can be obtained by using blocks of variable sizes with the FIBME algorithm. It should be noticed that the effectiveness of this feature depends on the complexity of the scene to be encoded. The more complex the motion, the highest the benefit of using blocks of variable size would be. For reasons of space, we report the results for Foreman and Football. The other sequences are characterized by a simpler motion, therefore the coding gain tends to be more limited. In all of the remaining simulations, blocks of variable sizes are always used.
TABLE 1: Normalized encoding time.

\begin{tabular}{lccc}
\hline Sequence & MC-EZBC & Full search & FIBME \\
\hline Silent & 1.35 & 19.32 & 1 \\
Mobile \& Calendar & 1.80 & 18.03 & 1 \\
Foreman & 1.60 & 18.56 & 1 \\
Football & 1.72 & 19.02 & 1 \\
City & 1.54 & 18.47 & 1 \\
Soccer & 1.60 & 19.14 & 1 \\
\hline
\end{tabular}

Figure 12 and Table 4 show the results of the simulations conducted when the scalable motion coding algorithm presented in Section 2.3 is introduced. In the $2 \mathrm{D}+\mathrm{t}$ codec, both FIBME and blocks of variable sizes are turned on. For the nonscalable case, motion vectors are encoded as in MCEZBC, by arithmetic coding the prediction residue obtained as the difference between the motion vector and its causal predictor. We can conclude the following:

(i) when the motion information is scaled, it is possible to achieve a lower target bitrate. In Figure 12, it can be noticed that in the nonscalable case, for the Football sequence, the minimum target bitrate is equal to $128 \mathrm{kbps}$, whereas it is possible to decode at $64 \mathrm{kbps}$ when the proposed algorithm is used;

(ii) at low bitrates, a PSNR improvement (up to $+3 \mathrm{~dB}$ ) is obtained by scaling the motion information as a fraction of the bit budget can be allocated to encode wavelet residual coefficients;

(iii) at high bitrates, the proposed algorithm causes a limited coding efficiency loss (less than $0.1 \mathrm{~dB}$ ) due to the fact that the lossless representation produced in the scalable case is not as efficient as in the nonscalable case. This is the cost associated to the embedded representation of the motion information.

Finally, we discuss the effect of truncating the motion vectors when a sequence is decoded at reduced spatial resolutions. In this case, there is no unique reference for the PSNR computation [33], since the truncation of motion vectors affects the MCTF synthesis phase. For this reason, in Figure 13 and Table 5, PSNR values are computed using the spatially downsampled sequence obtained extracting the $L L^{K}$ subband from the wavelet spatial analysis. When the sequences are decoded at reduced frame rate, the frame skipped sequence is used as a reference. Experimental subjective results [33] demonstrated that the relationship between visual quality and objective PSNR measurements is usually weak, unless the reference stays the same. For this reason, we also carried out visual tests in order to assess the effect of motion vectors truncation in terms of visual quality. In Figure 14, it is possible to see a sample frame from the Mobile \& Calendar sequence where it is clearly visible that motion vector truncation affects the visual quality of the reconstructed sequence. The same evidence is also true for all of the other tested sequences.

As a final remark, we can see that the proposed codec has a coding efficiency comparable with the $\mathrm{t}+2 \mathrm{D} \mathrm{MC}-\mathrm{EZBC}$ codec at full resolution and tends to outperform MC-EZBC 

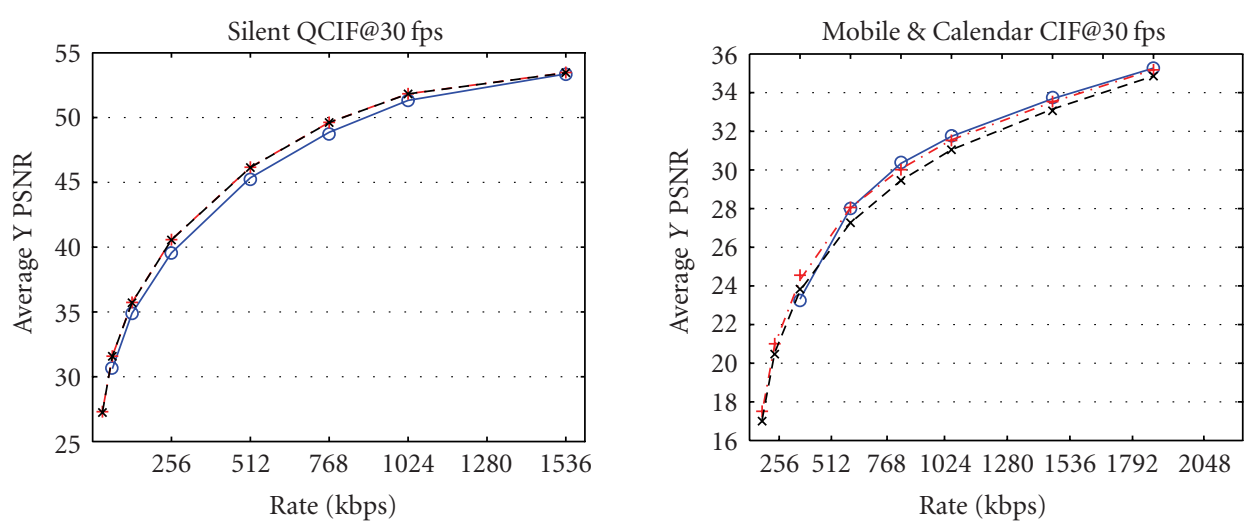

$-\mathrm{t}+2 \mathrm{D}(\mathrm{MC}-\mathrm{EZBC})$
$+-2 \mathrm{D}+\mathrm{t}-$ full search
$-*-2 \mathrm{D}+\mathrm{t}-$ FIBME

(a)

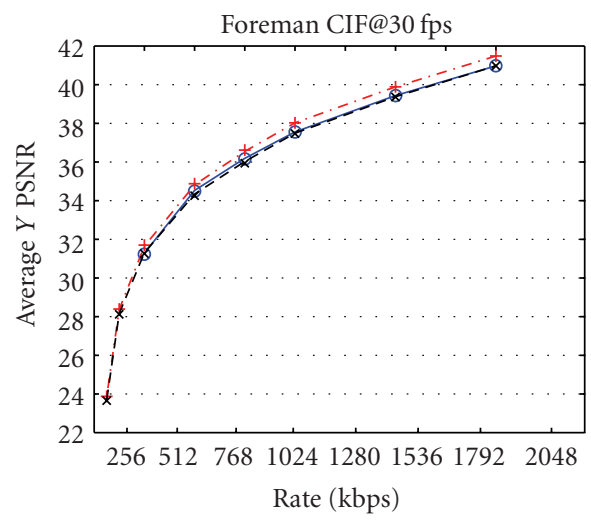

- $\mathrm{t}+2 \mathrm{D}(\mathrm{MC}-\mathrm{EZBC})$

- + 2D+t-full search

-* $-2 \mathrm{D}+\mathrm{t}-\mathrm{FIBME}$

(c)

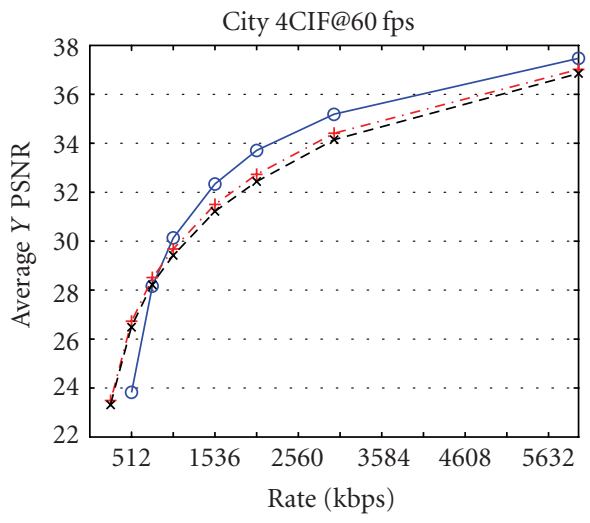

$-\mathrm{t}+2 \mathrm{D}(\mathrm{MC}-\mathrm{EZBC})$

$\cdot-2 \mathrm{D}+\mathrm{t}-$ full search

- * $-2 \mathrm{D}+\mathrm{t}-\mathrm{FIBME}$

(e)
- $\mathrm{t}+2 \mathrm{D}$ (MC-EZBC)

- $+-2 \mathrm{D}+\mathrm{t}$-full search

$-*-2 \mathrm{D}+\mathrm{t}-\mathrm{FIBME}$

(b)

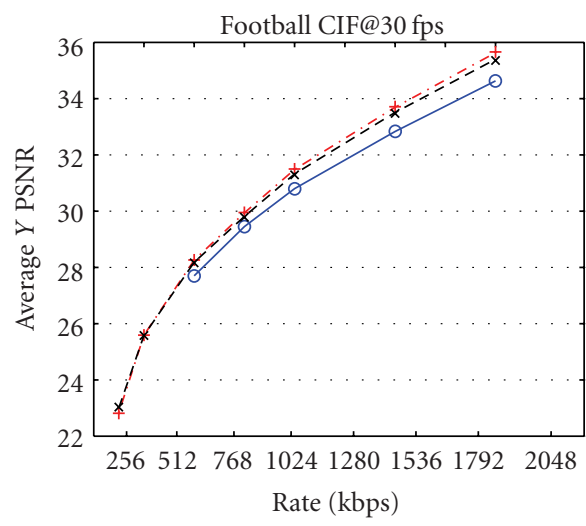

- $\mathrm{t}+2 \mathrm{D}$ (MC-EZBC)

$+-2 \mathrm{D}+\mathrm{t}-$ full search

-* $2 \mathrm{D}+\mathrm{t}-\mathrm{FIBME}$

(d)

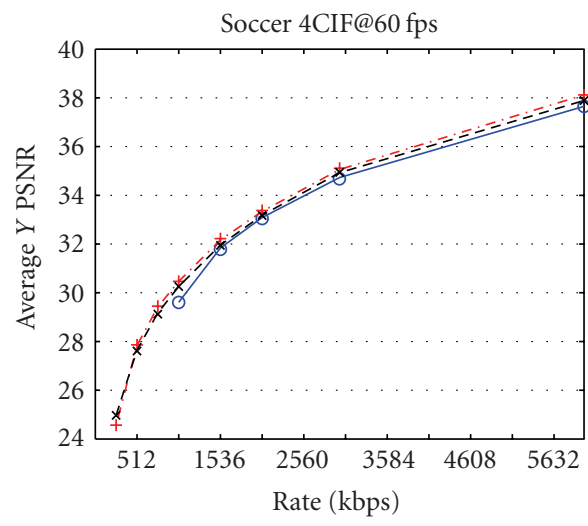

$\multimap \mathrm{t}+2 \mathrm{D}(\mathrm{MC}-\mathrm{EZBC})$

$+-2 \mathrm{D}+\mathrm{t}-$ full search

$-*-2 \mathrm{D}+\mathrm{t}-\mathrm{FIBME}$

(f)

Figure 10: FIBME versus full search. 
TABLE 2: Comparison between MC-EZBC ( $\mathrm{t}+2 \mathrm{D}$ with HVSBM), 2D+t with full search motion estimation, and 2D+t with FIBME.

(a) Silent QCIF@30 fps

\begin{tabular}{cccccc}
\hline kbps & $\begin{array}{c}\text { MC- } \\
\text { EZBC (a) }\end{array}$ & $\begin{array}{c}2 \mathrm{D}+\mathrm{t} \\
\text { FS (b) }\end{array}$ & $\begin{array}{c}2 \mathrm{D}+\mathrm{t} \\
\text { FIBME (c) }\end{array}$ & (c)-(a) & (c)-(b) \\
\hline 32 & - & 27.30 & 27.25 & - & -0.05 \\
64 & 30.61 & 31.59 & 31.58 & 0.97 & -0.01 \\
128 & 34.90 & 35.73 & 35.71 & 0.81 & -0.02 \\
256 & 39.56 & 40.58 & 40.56 & 1.00 & -0.02 \\
512 & 45.35 & 46.17 & 46.15 & 0.80 & -0.02 \\
768 & 48.87 & 49.63 & 49.62 & 0.75 & -0.01 \\
1024 & 51.33 & 51.84 & 51.83 & 0.50 & -0.01 \\
1536 & 53.37 & 53.47 & 53.46 & 0.09 & -0.01 \\
\hline
\end{tabular}

(c) Foreman CIF@30 fps

\begin{tabular}{cccccc}
\hline kbps & $\begin{array}{c}\text { MC- } \\
\text { EZBC (a) }\end{array}$ & $\begin{array}{c}2 \mathrm{D}+\mathrm{t} \\
\text { FS (b) }\end{array}$ & $\begin{array}{c}2 \mathrm{D}+\mathrm{t} \\
\text { FIBME (c) }\end{array}$ & (c)-(a) & (c)-(b) \\
\hline 64 & - & 23.86 & 23.69 & - & -0.17 \\
128 & - & 28.37 & 28.16 & - & -0.21 \\
256 & 31.29 & 31.65 & 31.31 & 0.02 & -0.34 \\
512 & 34.53 & 34.82 & 34.34 & -0.19 & -0.48 \\
768 & 36.19 & 36.55 & 36.03 & -0.16 & -0.52 \\
1024 & 37.56 & 38.03 & 37.49 & -0.07 & -0.54 \\
1536 & 39.44 & 39.89 & 39.38 & -0.06 & -0.51 \\
2048 & 40.98 & 41.49 & 40.98 & 0 & -0.51 \\
\hline
\end{tabular}

(e) City4CIF@60 fps

\begin{tabular}{cccccc}
\hline \multirow{2}{*}{ kbps } & $\begin{array}{c}\text { MC- } \\
\text { EZBC (a) }\end{array}$ & $\begin{array}{c}\text { 2D+t } \\
\text { FS (b) }\end{array}$ & $\begin{array}{c}\text { 2D+t } \\
\text { FIBME (c) }\end{array}$ & (c)-(a) & (c)-(b) \\
\hline 256 & - & 23.48 & 23.34 & - & -0.14 \\
512 & 23.82 & 26.71 & 26.52 & 2.70 & -0.19 \\
768 & 28.16 & 28.49 & 28.26 & 0.10 & -0.23 \\
1024 & 30.13 & 29.68 & 29.42 & -0.71 & -0.26 \\
1536 & 32.34 & 31.50 & 31.22 & -1.12 & -0.28 \\
2048 & 33.71 & 32.73 & 32.44 & -1.27 & -0.29 \\
3000 & 35.19 & 34.41 & 34.15 & -1.04 & -0.26 \\
6000 & 37.47 & 37.02 & 36.85 & -0.62 & -0.17 \\
\hline
\end{tabular}

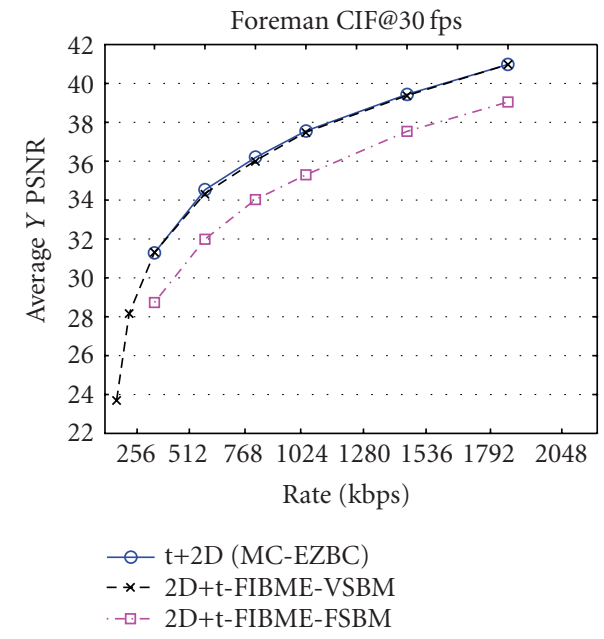

(a) (b) Mobile \& Calendar CIF@30 fps

\begin{tabular}{cccccc}
\hline kbps & $\begin{array}{c}\text { MC- } \\
\text { EZBC (a) }\end{array}$ & $\begin{array}{c}\text { FS (b) } \\
\text { FI }\end{array}$ & $\begin{array}{c}2 \mathrm{D}+\mathrm{t} \\
\text { FIBME (c) }\end{array}$ & (c)-(a) & (c)-(b) \\
\hline 64 & - & 17.46 & 17.07 & - & -0.39 \\
128 & - & 20.95 & 20.54 & - & -0.41 \\
256 & 23.31 & 24.31 & 23.76 & 0.45 & -0.55 \\
512 & 28.02 & 28.05 & 27.27 & -0.75 & -0.78 \\
768 & 30.34 & 30.06 & 29.46 & -0.88 & -0.60 \\
1024 & 31.74 & 31.56 & 31.05 & -0.69 & -0.51 \\
1536 & 33.69 & 33.52 & 33.15 & -0.54 & -0.37 \\
2048 & 35.27 & 35.19 & 34.86 & -0.41 & -0.33 \\
\hline
\end{tabular}

(d) Football CIF@30 fps

\begin{tabular}{cccccc}
\hline kbps & $\begin{array}{c}\text { MC- } \\
\text { EZBC (a) }\end{array}$ & $\begin{array}{c}2 \mathrm{D}+\mathrm{t} \\
\text { FS (b) }\end{array}$ & $\begin{array}{c}2 \mathrm{D}+\mathrm{t} \\
\text { FIBME (c) }\end{array}$ & (c)-(a) & (c)-(b) \\
\hline 64 & - & - & - & - & - \\
128 & - & 22.83 & 23.00 & - & 0.17 \\
256 & - & 25.59 & 25.58 & - & -0.01 \\
512 & 27.70 & 28.26 & 28.18 & 0.48 & -0.08 \\
768 & 29.45 & 29.94 & 29.81 & 0.36 & -0.13 \\
1024 & 30.80 & 31.48 & 31.32 & 0.52 & -0.16 \\
1536 & 32.83 & 33.69 & 33.51 & 0.68 & -0.18 \\
2048 & 34.63 & 35.63 & 35.40 & 0.77 & -0.23 \\
\hline
\end{tabular}

(f) Soccer4CIF@60 fps

\begin{tabular}{cccccr}
\hline kbps & $\begin{array}{c}\text { MC- } \\
\text { EZBC (a) }\end{array}$ & $\begin{array}{c}\text { FS (b) } \\
\text { FI }\end{array}$ & $\begin{array}{c}2 \mathrm{D}+\mathrm{t} \\
\text { FIBME (c) }\end{array}$ & (c)-(a) & (c)-(b) \\
\hline 256 & - & 24.57 & 24.97 & - & 0.40 \\
512 & - & 27.84 & 27.65 & - & -0.19 \\
768 & - & 29.42 & 29.17 & - & -0.25 \\
1024 & 29.61 & 30.47 & 30.26 & 0.65 & -0.21 \\
1536 & 31.84 & 32.17 & 31.96 & 0.12 & -0.21 \\
2048 & 33.09 & 33.34 & 33.18 & 0.09 & -0.16 \\
3000 & 34.73 & 35.06 & 34.93 & 0.20 & -0.13 \\
6000 & 37.65 & 38.12 & 37.89 & 0.24 & -0.23 \\
\hline
\end{tabular}

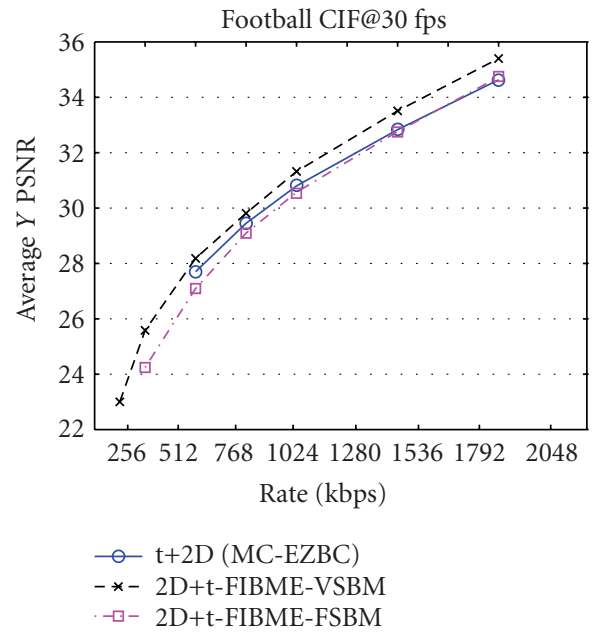

(b)

FIgURE 11: FIBME: fixed block sizes versus variable block sizes. 
TABLE 3: FIBME: fixed block sizes (FSBM) versus variable block sizes (VSBM).

(a) Foreman CIF@30 fps

\begin{tabular}{cccccc}
\hline \multirow{2}{*}{ kbps } & $\begin{array}{c}\text { MC- } \\
\text { EZBC (a) }\end{array}$ & $\begin{array}{c}\text { 2D+t FIBME } \\
\text { FSBM (b) }\end{array}$ & $\begin{array}{c}\text { VSBM (c) } \\
\text { VSBMM }\end{array}$ & (c)-(a) & (c)-(b) \\
\hline 64 & - & - & 23.69 & - & - \\
128 & - & - & 28.16 & - & - \\
256 & 31.29 & 28.73 & 31.31 & 0.02 & 2.58 \\
512 & 34.53 & 31.99 & 34.34 & -0.19 & 2.35 \\
768 & 36.19 & 34.03 & 36.03 & -0.16 & 2.00 \\
1024 & 37.56 & 35.30 & 37.49 & -0.07 & 2.19 \\
1536 & 39.44 & 37.54 & 39.38 & -0.06 & 1.84 \\
2048 & 40.98 & 39.05 & 40.98 & 0 & 1.93 \\
\hline
\end{tabular}

(b) Football CIF@30 fps

\begin{tabular}{|c|c|c|c|c|c|}
\hline kbps & $\begin{array}{c}\text { MC- } \\
\text { EZBC (a) }\end{array}$ & $\begin{array}{c}\text { 2D+t FIBME } \\
\text { FSBM (b) }\end{array}$ & $\begin{array}{c}2 \mathrm{D}+\mathrm{t} \text { FIBME } \\
\operatorname{VSBM}(\mathrm{c})\end{array}$ & (c) $-(\mathrm{a})$ & (c) $-(\mathrm{b})$ \\
\hline 64 & - & - & - & - & - \\
\hline 128 & - & - & 23.00 & - & - \\
\hline 256 & - & 24.24 & 25.58 & - & 1.34 \\
\hline 512 & 27.70 & 27.09 & 28.18 & 0.48 & 0.72 \\
\hline 1024 & 30.80 & 30.57 & 31.32 & 0.52 & 0.75 \\
\hline 1536 & 32.83 & 32.76 & 33.51 & 0.68 & 0.75 \\
\hline 2048 & 34.63 & 34.73 & 35.40 & 0.77 & 0.67 \\
\hline
\end{tabular}

TABLE 4: Nonscalable versus scalable motion vectors.

(a) Silent QCIF@30 fps

\begin{tabular}{cccccr}
\hline kbps & $\begin{array}{c}\text { MC- } \\
\text { EZBC (a) }\end{array}$ & $\begin{array}{c}\text { Nonscal. } \\
\text { MV (b) }\end{array}$ & $\begin{array}{c}\text { Scal. } \\
\text { MV (c) }\end{array}$ & (c)-(a) & (c)-(b) \\
\hline 32 & - & 27.25 & 27.50 & - & 0.25 \\
64 & 30.61 & 31.58 & 31.70 & 1.09 & 0.12 \\
128 & 34.90 & 35.71 & 35.80 & 0.90 & 0.09 \\
256 & 39.56 & 40.56 & 40.60 & 1.04 & 0.04 \\
512 & 45.35 & 46.15 & 46.14 & 0.79 & -0.01 \\
768 & 48.87 & 49.62 & 49.60 & 0.73 & -0.02 \\
1024 & 51.33 & 51.83 & 51.79 & 0.46 & -0.04 \\
1536 & 53.37 & 53.46 & 53.40 & 0.03 & -0.06 \\
\hline
\end{tabular}

(c) Foreman CIF@30 fps

\begin{tabular}{cccccr}
\hline kbps & $\begin{array}{c}\text { MC- } \\
\text { EZBC (a) }\end{array}$ & $\begin{array}{c}\text { Nonscal. } \\
\text { MV (b) }\end{array}$ & $\begin{array}{c}\text { Scal. } \\
\text { MV (c) }\end{array}$ & (c)-(a) & (c)-(b) \\
\hline 64 & - & 23.69 & 25.80 & - & 2.11 \\
128 & - & 28.16 & 29.27 & - & 1.11 \\
256 & 31.29 & 31.31 & 32.01 & 0.72 & 0.70 \\
512 & 34.53 & 34.34 & 34.40 & -0.13 & 0.06 \\
768 & 36.19 & 36.03 & 36.02 & -0.17 & -0.01 \\
1024 & 37.56 & 37.49 & 37.47 & -0.09 & -0.02 \\
1536 & 39.44 & 39.38 & 39.33 & -0.11 & -0.05 \\
2048 & 40.98 & 40.98 & 40.93 & -0.05 & -0.05 \\
\hline
\end{tabular}

(e) City4CIF@60fps

\begin{tabular}{cccccr}
\hline kbps & $\begin{array}{c}\text { MC- } \\
\text { EZBC (a) }\end{array}$ & $\begin{array}{c}\text { Nonscal. } \\
\text { MV (b) }\end{array}$ & $\begin{array}{c}\text { Scal. } \\
\text { MV (c) }\end{array}$ & (c)-(a) & (c)-(b) \\
\hline 256 & - & 23.34 & 25.80 & - & 2.46 \\
512 & 23.82 & 26.52 & 27.82 & 4.00 & 1.30 \\
768 & 28.16 & 28.26 & 28.88 & 0.72 & 0.62 \\
1024 & 30.13 & 29.42 & 29.70 & -0.43 & 0.28 \\
1536 & 32.34 & 31.22 & 31.20 & -1.14 & -0.02 \\
2048 & 33.71 & 32.44 & 32.40 & -1.31 & -0.04 \\
3000 & 35.19 & 34.15 & 34.09 & -1.10 & -0.06 \\
6000 & 37.47 & 36.85 & 36.78 & -0.69 & -0.07 \\
\hline
\end{tabular}

(b) Mobile \& Calendar CIF@30 fps

\begin{tabular}{cccccr}
\hline kbps & $\begin{array}{c}\text { MC- } \\
\text { EZBC (a) }\end{array}$ & $\begin{array}{c}\text { Nonscal. } \\
\text { MV (b) }\end{array}$ & $\begin{array}{c}\text { Scal. } \\
\text { MV (c) }\end{array}$ & (c)-(a) & (c)-(b) \\
\hline 64 & - & 17.07 & 20.01 & - & 2.94 \\
128 & - & 20.54 & 22.10 & - & 1.56 \\
256 & 23.31 & 23.76 & 24.90 & 1.59 & 1.14 \\
512 & 28.02 & 27.27 & 27.60 & -0.42 & 0.33 \\
768 & 30.34 & 29.46 & 29.45 & -0.89 & -0.01 \\
1024 & 31.74 & 31.05 & 31.02 & -0.72 & -0.03 \\
1536 & 33.69 & 33.15 & 33.10 & -0.59 & -0.05 \\
2048 & 35.27 & 34.86 & 34.80 & -0.47 & -0.06 \\
\hline
\end{tabular}

(d) Football CIF@30 fps

\begin{tabular}{cccccc}
\hline kbps & $\begin{array}{c}\text { MC- } \\
\text { EZBC (a) }\end{array}$ & $\begin{array}{c}\text { Nonscal. } \\
\text { MV (b) }\end{array}$ & $\begin{array}{c}\text { Scal. } \\
\text { MV (c) }\end{array}$ & (c)-(a) & (c)-(b) \\
\hline 64 & - & - & 24.30 & - & - \\
128 & - & 23.00 & 25.30 & - & 2.30 \\
256 & - & 25.58 & 26.80 & - & 1.22 \\
512 & 27.70 & 28.18 & 28.80 & 1.10 & 0.62 \\
768 & 29.45 & 29.81 & 30.10 & 0.65 & 0.29 \\
1024 & 30.80 & 31.32 & 31.32 & 0.52 & 0.00 \\
1536 & 32.83 & 33.51 & 33.49 & 0.66 & -0.02 \\
2048 & 34.63 & 35.40 & 35.35 & 0.72 & -0.05 \\
\hline
\end{tabular}

(f) Soccer4CIF@60 fps

\begin{tabular}{cccccr}
\hline kbps & $\begin{array}{c}\text { MC- } \\
\text { EZBC (a) }\end{array}$ & $\begin{array}{c}\text { Nonscal. } \\
\text { MV (b) }\end{array}$ & $\begin{array}{c}\text { Scal. } \\
\text { MV (c) }\end{array}$ & (c)-(a) & (c)-(b) \\
\hline 256 & - & 24.97 & 26.50 & - & 1.53 \\
512 & - & 27.65 & 28.75 & - & 1.10 \\
768 & - & 29.17 & 29.90 & - & 0.73 \\
1024 & 29.61 & 30.26 & 30.80 & 1.19 & 0.54 \\
1536 & 31.84 & 31.96 & 32.03 & 0.19 & 0.07 \\
2048 & 33.09 & 33.18 & 33.15 & 0.06 & -0.03 \\
3000 & 34.73 & 34.93 & 34.90 & 0.17 & -0.03 \\
6000 & 37.65 & 37.89 & 37.85 & 0.20 & -0.04 \\
\hline
\end{tabular}



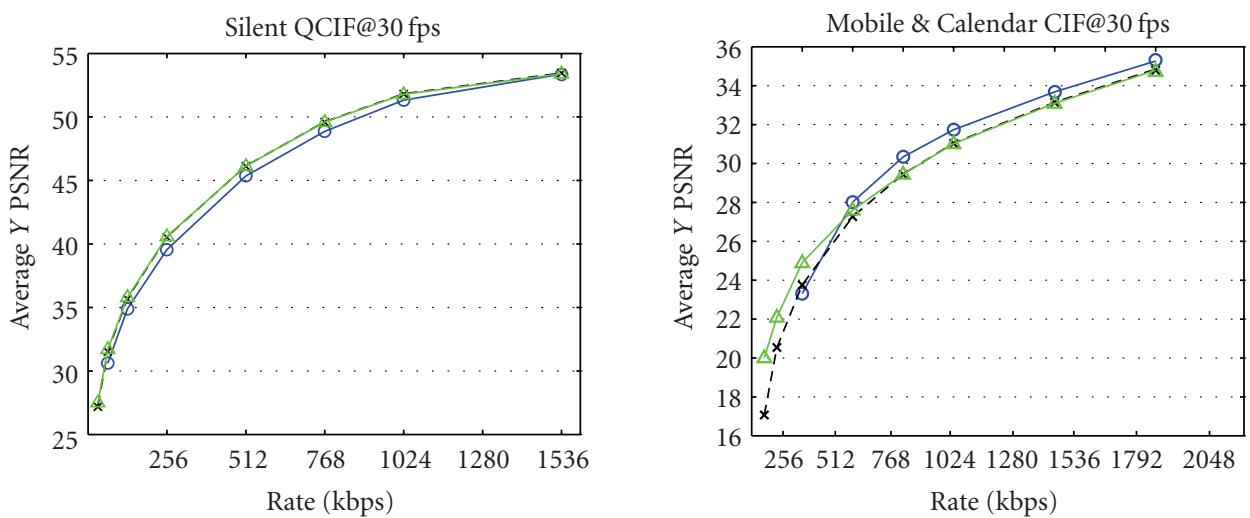

\section{- $\mathrm{t}+2 \mathrm{D}(\mathrm{MC}-\mathrm{EZBC})$ \\ - * - 2D+t-nonscalable MV \\ $\triangle$ 2D+t-scalable MV}

(a)

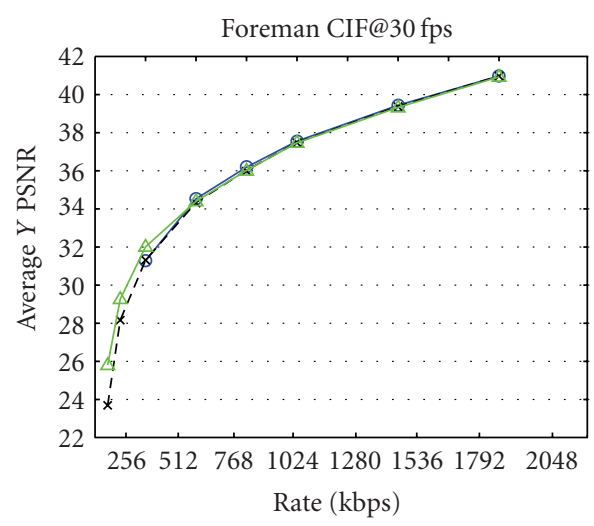

$-\mathrm{t}+2 \mathrm{D}(\mathrm{MC}-\mathrm{EZBC})$

- * - 2D+t-nonscalable MV

$\triangle$ 2D+t-scalable MV

(c)

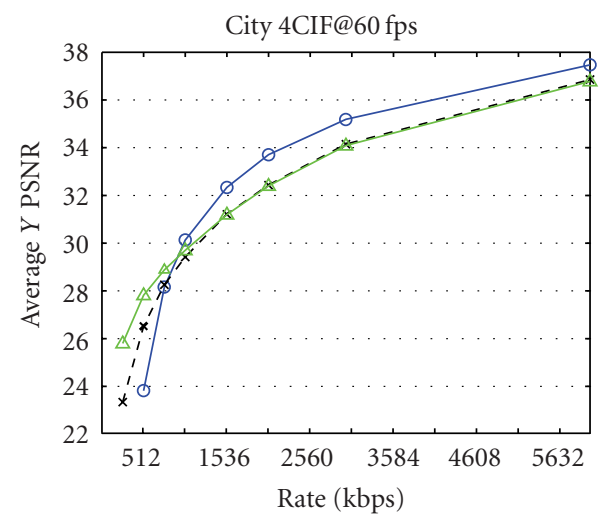

$\multimap \mathrm{t}+2 \mathrm{D}(\mathrm{MC}-\mathrm{EZBC})$

-* - 2D+t-nonscalable MV

$\triangle$ 2D+t-scalable MV

(e) $\multimap \mathrm{t}+2 \mathrm{D}(\mathrm{MC}-\mathrm{EZBC})$

-* - 2D+t-nonscalable MV

$\triangle$ 2D+t-scalable MV

(b)

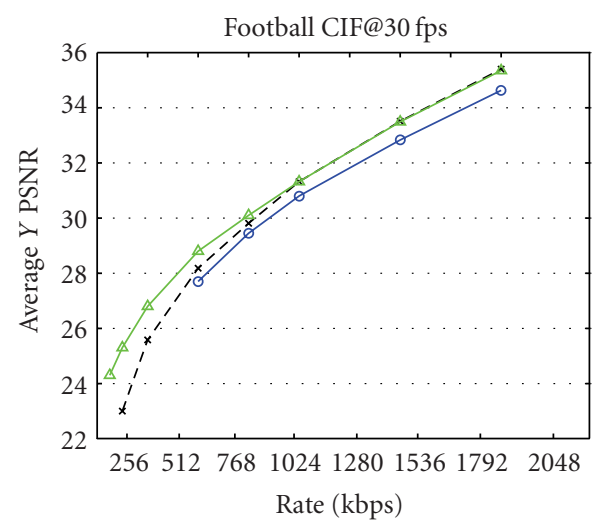

$\rightarrow \mathrm{t}+2 \mathrm{D}(\mathrm{MC}-\mathrm{EZBC})$

- * $-2 \mathrm{D}+\mathrm{t}-$ nonscalable MV

$\triangle$ 2D+t-scalable MV

(d)

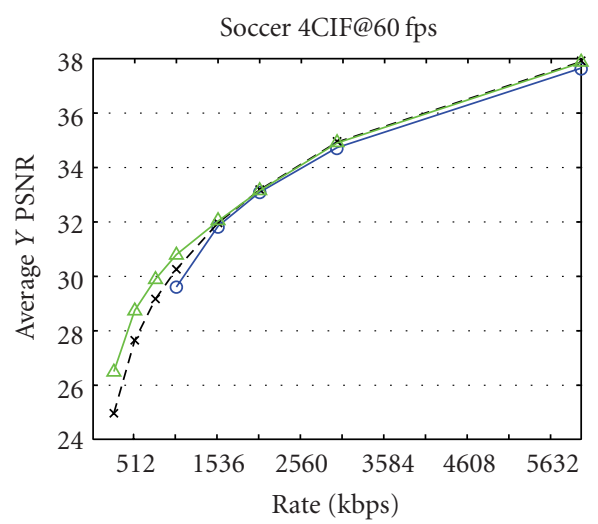

$\rightarrow \mathrm{t}+2 \mathrm{D}(\mathrm{MC}-\mathrm{EZBC})$

-* $-2 \mathrm{D}+\mathrm{t}-$ nonscalable MV

$\triangle$ 2D+t-scalable MV

(f)

Figure 12: Nonscalable versus scalable motion vectors. 

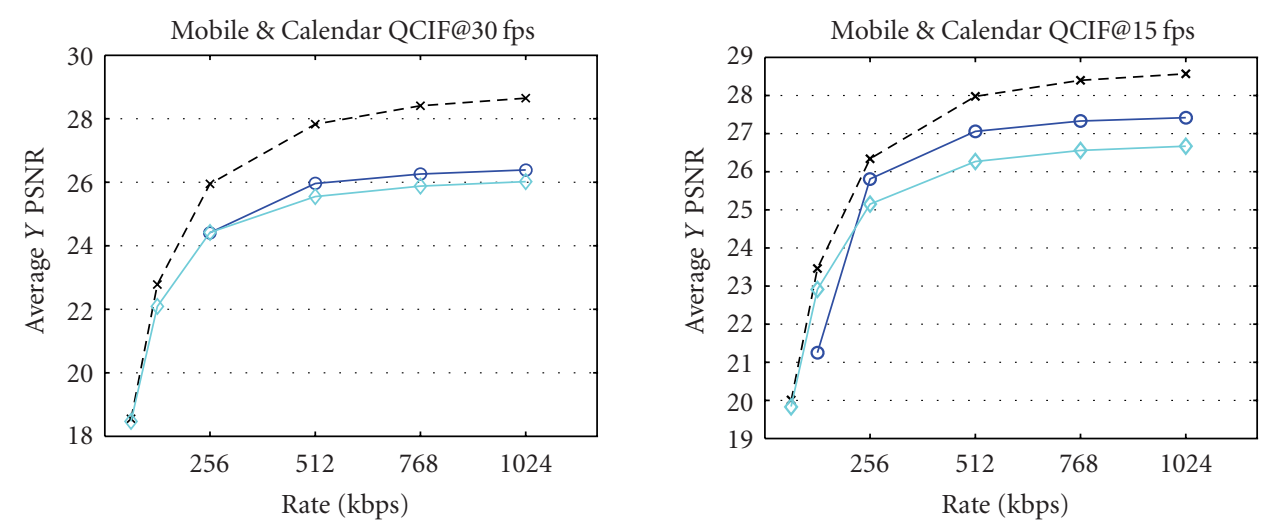

$$
\begin{aligned}
& -\mathrm{t}+2 \mathrm{D}(\mathrm{MC}-\mathrm{EZBC}) \\
& -*-2 \mathrm{D}+\mathrm{t}-\text { no MV truncation } \\
& -2 \mathrm{D}+\mathrm{t}-\mathrm{MV} \text { truncation }
\end{aligned}
$$

(a)

$$
\begin{aligned}
& -\mathrm{t}+2 \mathrm{D}(\mathrm{MC}-\mathrm{EZBC}) \\
& -*-2 \mathrm{D}+\mathrm{t}-\text { no MV truncation } \\
& --2 \mathrm{D}+\mathrm{t}-\mathrm{MV} \text { truncation }
\end{aligned}
$$

(b)

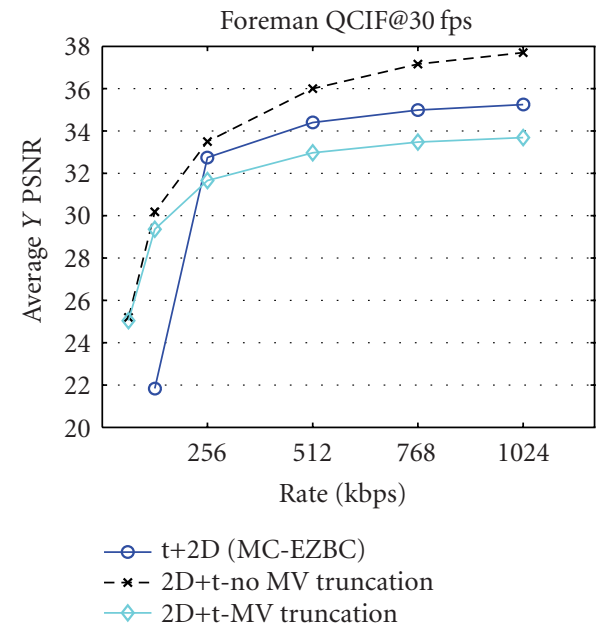

(c)

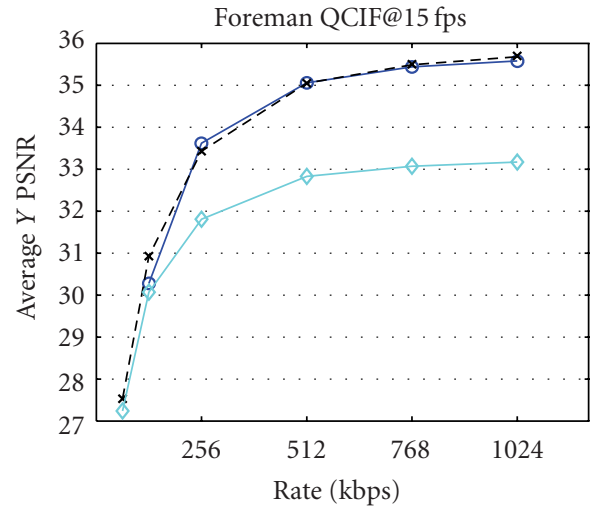

$-\mathrm{t}+2 \mathrm{D}(\mathrm{MC}-\mathrm{EZBC})$

- * - 2D+t-no MV truncation

$\rightarrow-2 \mathrm{D}+\mathrm{t}-\mathrm{MV}$ truncation

(d)

FIGURE 13: Effect of motion vector truncation.

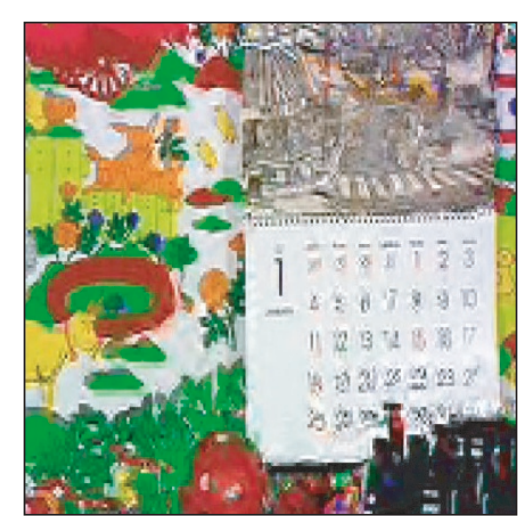

(a)

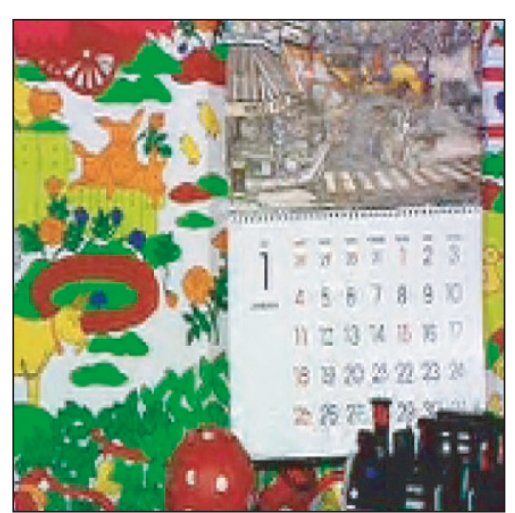

(b)

FIGURE 14: Effect of motion vector truncation. Mobile \& Calendar, QCIF@30 fps—256 kbps: (a) with MV truncation; (b) without MV truncation. 
TABLE 5: FIBME: fixed block sizes (FSBM) versus variable block sizes (VSBM).

(a) Mobile QCIF@30 fps

\begin{tabular}{cccccc}
\hline kbps & $\begin{array}{c}\text { MC- } \\
\text { EZBC (a) }\end{array}$ & $\begin{array}{c}\text { MV } \\
\text { trunc. (b) }\end{array}$ & $\begin{array}{c}\text { No MV } \\
\text { trunc. (c) }\end{array}$ & (c)-(a) & (c)-(b) \\
\hline 64 & - & 18.47 & 18.53 & - & 0.06 \\
128 & - & 22.09 & 22.78 & - & 0.69 \\
256 & 24.41 & 24.42 & 25.95 & 1.54 & 1.53 \\
512 & 25.96 & 25.55 & 27.83 & 1.87 & 2.28 \\
768 & 26.26 & 25.88 & 28.41 & 2.15 & 2.53 \\
1024 & 26.39 & 26.02 & 28.65 & 2.26 & 2.63 \\
\hline
\end{tabular}

(c) Foreman QCIF@30 fps

\begin{tabular}{cccccc}
\hline kbps & $\begin{array}{c}\text { MC- } \\
\text { EZBC (a) }\end{array}$ & $\begin{array}{c}\text { MV } \\
\text { trunc. (b) }\end{array}$ & $\begin{array}{c}\text { No MV } \\
\text { trunc. (c) }\end{array}$ & (c)-(a) & (c)-(b) \\
\hline 64 & - & 25.04 & 25.16 & - & 0.12 \\
128 & 21.84 & 29.36 & 30.18 & 8.34 & 0.82 \\
256 & 32.75 & 31.66 & 33.50 & 0.75 & 1.84 \\
512 & 34.40 & 32.97 & 36 & 1.60 & 3.03 \\
768 & 34.99 & 33.48 & 37.16 & 2.17 & 3.68 \\
1024 & 35.25 & 33.69 & 37.70 & 2.45 & 4.01 \\
\hline
\end{tabular}

at reduced spatio-temporal resolution. Furthermore, the sequences decoded with the proposed $2 \mathrm{D}+\mathrm{t}$ codec are not affected by blocking artifacts that appear in the MC-EZBC codec, thus improving the subjective quality when they attain the same objective performance.

\section{CONCLUSIONS}

In this paper, we present a fully scalable $2 \mathrm{D}+\mathrm{t}$ video codec, where we introduced novel algorithms in the motion estimation and signaling part. The proposed fast motion estimation algorithm is able to achieve good coding efficiency at a fraction of the computational complexity of a full search approach. The scalable representation of motion information improves the objective and subjective quality of sequences decoded at low bitrates. Our current research activities in this area focus on the study of rate-distortion optimal motion modeling in wavelet-based $2 \mathrm{D}+\mathrm{t}$ video coding schemes.

\section{REFERENCES}

[1] D. Taubam and M. W. Marcellin, JPEG2000: Image Compression Fundamentals, Standards and Practice, Kluwer Academic, Boston, Mass, USA, 2002.

[2] A. Said and W. A. Pearlman, "A new, fast, and efficient image codec based on set partitioning in hierarchical trees," IEEE Transactions on Circuits and Systems for Video Technology, vol. 6, no. 3, pp. 243-250, 1996.

[3] S.-T. Hsiang and J. W. Woods, "Embedded image coding using zeroblocks of subband/wavelet coefficients and context modeling," in Proceedings of IEEE International Symposium on Circuits and Systems (ISCAS '00), vol. 3, pp. 662-665, Geneva, Switzerland, May 2000. (b) Mobile QCIF@15 fps

\begin{tabular}{cccccc}
\hline kbps & $\begin{array}{c}\text { MC- } \\
\text { EZBC (a) }\end{array}$ & $\begin{array}{c}\text { MV } \\
\text { trunc. (b) }\end{array}$ & $\begin{array}{c}\text { No MV } \\
\text { trunc. (c) }\end{array}$ & (c)-(a) & (c)-(b) \\
\hline 64 & - & 19.84 & 19.98 & - & 0.14 \\
128 & 21.25 & 22.91 & 23.46 & 2.21 & 0.55 \\
256 & 25.81 & 25.15 & 26.34 & 0.53 & 1.19 \\
512 & 27.06 & 26.27 & 27.98 & 0.92 & 1.71 \\
768 & 27.33 & 26.56 & 28.40 & 1.07 & 1.84 \\
1024 & 27.42 & 26.67 & 28.57 & 1.15 & 1.90 \\
\hline
\end{tabular}

(d) Foreman QCIF@15 fps

\begin{tabular}{cccccc}
\hline kbps & $\begin{array}{c}\text { MC- } \\
\text { EZBC (a) }\end{array}$ & $\begin{array}{c}\text { MV } \\
\text { trunc. (b) }\end{array}$ & $\begin{array}{c}\text { No MV } \\
\text { trunc. (c) }\end{array}$ & (c)-(a) & (c)-(b) \\
\hline 64 & - & 27.24 & 27.53 & - & 0.29 \\
128 & 30.28 & 30.07 & 30.93 & 0.65 & 0.86 \\
256 & 33.62 & 31.81 & 33.43 & -0.19 & 1.62 \\
512 & 35.06 & 32.83 & 35.05 & -0.01 & 2.22 \\
768 & 35.44 & 33.07 & 35.49 & 0.05 & 2.42 \\
1024 & 35.58 & 33.17 & 35.68 & 0.10 & 2.51 \\
\hline
\end{tabular}

[4] J. Xu, Z. Xiong, S. Li, and Y.-Q. Zhang, “Three-dimensional embedded subband coding with optimized truncation (3D ESCOT)," Applied and Computational Harmonic Analysis, vol. 10, no. 3, pp. 290-315, 2001.

[5] P. Chen and J. W. Woods, "Bidirectional MC-EZBC with lifting implementation," IEEE Transactions on Circuits and Systems for Video Technology, vol. 14, no. 10, pp. 1183-1194, 2004.

[6] A. Secker and D. Taubman, "Lifting-based invertible motion adaptive transform (LIMAT) framework for highly scalable video compression," IEEE Transactions on Image Processing, vol. 12, no. 12, pp. 1530-1542, 2003.

[7] G. Pau, C. Tillier, B. Pesquet-Popescu, and H. Heijmans, "Motion compensation and scalability in lifting-based video coding," Signal Processing: Image Communication, vol. 19, no. 7, pp. 577-600, 2004.

[8] J.-R. Ohm, "Three-dimensional subband coding with motion compensation," IEEE Transactions on Image Processing, vol. 3, no. 5, pp. 559-571, 1994.

[9] Y. Andreopoulos, A. Munteanu, J. Barbarien, M. van der Schaar, J. Cornelis, and P. Schelkens, "In-band motion compensated temporal filtering," Signal Processing: Image Communication, vol. 19, no. 7, pp. 653-673, 2004.

[10] Y. Wang, S. Cui, and J. E. Fowler, "3D video coding using redundant-wavelet multihypothesis and motion-compensated temporal filtering," in Proceedings of IEEE International Conference on Image Processing (ICIP '03), vol. 2, pp. 755-758, Barcelona, Spain, September 2003.

[11] H.-W. Park and H.-S. Kim, "Motion estimation using lowband-shift method for wavelet-based moving-picture coding," IEEE Transactions on Image Processing, vol. 9, no. 4, pp. 577$587,2000$.

[12] J. C. Ye and M. van der Schaar, "Fully scalable 3D overcomplete wavelet video coding using adaptive motion compensated temporal filtering," in Visual Communications and Image Processing (VCIP '03), T. Ebrahimi and T. Sikora, Eds., vol. 5150 of 
Proceedings of SPIE, pp. 1169-1180, Lugano, Switzerland, July 2003.

[13] S. Mallat, A Wavelet Tour of Signal Processing, Academic Press, San Diego, Calif, USA, 1998.

[14] N. Mehrseresht and D. Taubman, "A flexible structure for fully scalable motion compensated 3D-DWT with emphasis on the impact of spatial scalability," submitted to IEEE Transactions on Image Processing.

[15] N. Mehrseresht and D. Taubman, "An efficient contentadaptive motion compensated 3D-DWT with enhanced spatial and temporal scalability," submitted to IEEE Transactions on Image Processing.

[16] ITU-T, Information technology-Coding of audio-visual objects_Part 10: Advanced video coding, May 2003, ISO/IEC International Standard 14496-10:2003.

[17] D. Maestroni, A. Sarti, M. Tagliasacchi, and S. Tubaro, "Fast in-band motion estimation with variable size block matching," in Proceedings of IEEE International Conference on Image Processing (ICIP '04), vol. 4, pp. 2287-2290, Singapore, October 2004.

[18] D. Maestroni, A. Sarti, M. Tagliasacchi, and S. Tubaro, "Scalable coding of variable size blocks motion vectors," in Proceedings of IEEE International Conference on Image Processing (ICIP '04), vol. 2, pp. 1333-1336, Singapore, October 2004.

[19] D. Maestroni, A. Sarti, M. Tagliasacchi, and S. Tubaro, "Wavelet-based video coding: optimal use of motion information for the decoding of spatially scaled video sequences," in Proceedings of the 12th European Signal Processing Conference (EUSIPCO '04), Vienna, Austria, September 2004.

[20] Y. Andreopoulos, A. Munteanu, G. Van der Auwera, P. Schelkens, and J. Cornelis, "A new method for complete-toovercomplete discrete wavelet transforms," in Proceedings of the 14th International Conference on Digital Signal Processing (DSP '02), vol. 2, pp. 501-504, Santorini, Greece, July 2002.

[21] Y. Andreopoulos, M. van der Schaar, A. Munteanu, J. Barbarien, P. Schelkens, and J. Cornelis, "Fully-scalable wavelet video coding using in-band motion compensated temporal filtering," in Proceedings of IEEE International Conference on Acoustics, Speech, and Signal Processing (ICASSP '03), vol. 3, pp. 417-420, Hong Kong, China, April 2003.

[22] W. Cai and M. Adjouadi, "An efficient approach of fast motion estimation and compensation in wavelet domain video compression," in Proceedings of IEEE International Conference on Acoustics, Speech, and Signal Processing (ICASSP '04), vol. 2, pp. 977-980, Montreal, Quebec, Canada, May 2004.

[23] S.-J. Choi and J. W. Woods, "Motion-compensated 3-D subband coding of video," IEEE Transactions on Image Processing, vol. 8, no. 2, pp. 155-167, 1999.

[24] R. Xiong, F. Wu, S. Li, Z. Xiong, and Y.-Q. Zhang, "Exploiting temporal correlation with adaptive block-size motion alignment for 3D wavelet coding," in Visual Communications and Image Processing 2004, vol. 5308 of Proceedings of SPIE, pp. 144-155, San Jose, Calif, USA, January 2004.

[25] D. Taubman and A. Secker, "Highly scalable video compression with scalable motion coding," in Proceedings of IEEE International Conference on Image Processing (ICIP '03), vol. 3, pp. 273-276, Barcelona, Spain, September 2003.

[26] A. Secker and D. Taubman, "Highly scalable video compression with scalable motion coding," IEEE Transactions on Image Processing, vol. 13, no. 8, pp. 1029-1041, 2004.

[27] J. Barbarien, A. Munteanu, F. Verdicchio, Y. Andreopoulos, J. Cornelis, and P. Schelkens, "Scalable motion vector coding," Electronics Letters, vol. 40, no. 15, pp. 932-934, 2004.
[28] G. Boisson, E. Francois, and C. Guillemot, "Accuracy-scalable motion coding for efficient scalable video compression," in Proceedings of IEEE International Conference on Image Processing (ICIP '04), vol. 2, pp. 1309-1312, Singapore, October 2004.

[29] R. Xiong, J. Xu, F. Wu, S. Li, and Y.-Q. Zhang, "Layered motion estimation and coding for fully scalable 3D wavelet video coding," in Proceeding of IEEE International Conference on Image Processing (ICIP '04), vol. 4, pp. 2271-2274, Singapore, October 2004.

[30] B. D. Lucas and T. Kanade, "An iterative image registration technique with an application to stereo vision," in Proceedings of the 7th International Joint Conference on Artificial Intelligence (IJCAI '81), pp. 674-679, Vancouver, BC, Canada, August 1981.

[31] B. K. P. Horn and B. G. Schunck, "Determining optical flow," Artificial Intelligence, vol. 17, no. 1-3, pp. 185-203, 1981.

[32] M. A. Tekalp, Digital Video Processing, Prentice-Hall Signal Processing Series, Prentice-Hall, Upper Saddle River, NJ, USA, 1995.

[33] U. Benzler and M. Wien, "Results of SVC CE3 (Quality Evaluation)," ISO/IEC JTC1/SC29/WG11 MPEG Document M10931, July 2004.

M. Tagliasacchi born in 1978, received the "Laurea" degree (2002, cum Laude) in computer systems engineering from Politecnico di Milano, Italy. He is currently working toward the Ph.D. degree at Politecnico di Milano. During 2004, he was Visiting Scholar at University of California at Berkeley. His research interests include distributed video coding, scalable video coding, wavelet-based video coding, and localization of acoustic sources.

D. Maestroni received the "Laurea" degree in communication systems engineering from Politecnico di Milano, Italy, in 2003. During 2004, he collaborated with Politecnico di Milano and Telecom Italia Lab in the research activities on scalable video coding within MPEG. His thesis work and further research activities concern waveletbased scalable video coding.
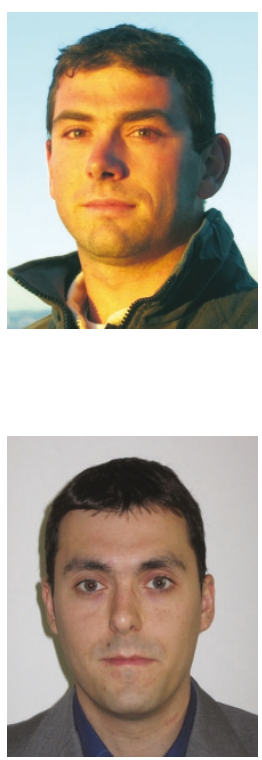

S. Tubaro born in 1957, completed his studies in electronic engineering at the "Politecnico di Milano," Italy, in 1982. He then joined the "Dipartimento di Elettronica e Informazione" of the "Politecnico di Milano," first as a Researcher of the National Research Council, then (in November 1991) as an Associate Professor, and from December 2004 as a Full Professor. In the first years of activities, he worked on problems

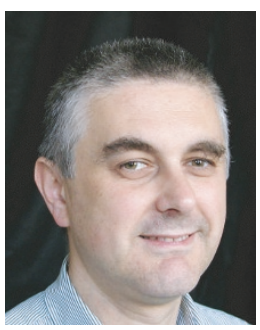
related to speech analysis; motion estimation/compensation for video analysis/coding, and vector quantization applied to hybrid video coding. In the past few years, his research interests have focused on image and video analysis for the geometric and radiometric modeling of 3D scenes, advanced algorithms for video coding and sound processing. He authored more than 150 scientific publications on international journals and congresses. He coauthored two books on digital processing of video sequences. He 
is also a co-author of several patents relative to image processing techniques. He coordinates the research activities of the image and sound processing group (ISPG) at the "Dipartimento di Elettronica e Informazione" of the "Politecnico di Milano" that is involved in several research programs funded by industrial partners, the Italian Government and by the European Commission.

A. Sarti born in 1963, received the "Laurea" degree (1988, cum Laude) and the Ph.D. (1993) in electrical engineering, from the University of Padua, Italy. He completed his graduate studies at the University of California at Berkeley. In 1993, he joined the Dipartimento di Elettronica e Informazione of the Politecnico di Milano, where he is now an Associate Professor. His current research interests are in the area of digital signal pro-

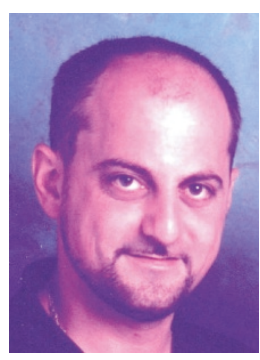
cessing, with particular focus on computer vision, image and sound processing. 